
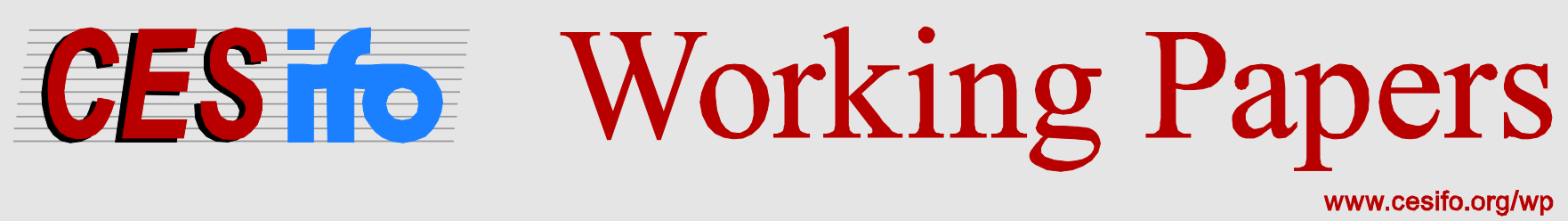

\title{
Distributional Preferences in Probabilistic and Share Contests
}

\author{
Magnus Hoffmann \\ Martin Kolmar
}

CESIFO WORKING PAPER NO. 4184

CATEGORY 2: Public CHOICE

APRIL 2013

An electronic version of the paper may be downloaded

- from the SSRN website:

- from the RePEc website:

- from the CESifo website:

WWW.SSRN.com

Www.RePEc.org

www.CESifo-group.org/wp

\section{CESifo}




\title{
Distributional Preferences in Probabilistic and Share Contests
}

\begin{abstract}
We analyze Nash equilibria of share and probabilistic contests when players have distributional preferences. If players are sufficiently similar, distributional preferences create multiple equilibria. For the case of only mildly heterogeneous players, equilibrium effort can be lower as well as higher than effort with standard, selfish preferences. These findings can explain three anomalies in empirical tests of the probabilistic prize-seeking game, namely the large variance of effort levels (overspreading), individual spending that exceeds the Nashequilibrium prediction (overspending), and aggregate spending that exceeds the value of the prize (overdissipation), and they are also in line with the findings for share contests. If players are sufficiently heterogeneous, the game has a unique equilibrium that is more egalitarian than the standard, selfish Nash equilibrium. It turns out that the less talented competitor may win the larger share of the prize if his inequality aversion is sufficiently strong. We analyze how the equilibria evolve if the number of players gets larger and how sequential moves influence behavior. Two new insights follow from the analysis of the sequential-move game. First, sequential moves act as a coordination device if there are multiple simultaneous equilibria, and second the inequality aversion of the more egalitarian player can be used as a commitment device for low effort. This effect can reverse the conventional wisdom that the underdog should lead.
\end{abstract}

JEL-Code: D030, D720, D740.

Keywords: contests, distributional preferences, overdissipation.

\author{
Magnus Hoffmann \\ School of Economics and Management \\ University of Technology \\ Straße des 17. Juni 135 \\ Germany-10623 Berlin \\ magnus.hoffmann@tu-berlin.de
}

\author{
Martin Kolmar \\ Institute of Economics \\ University of St. Gallen \\ Varnbüelstraße 19 \\ Switzerland -9000 St. Gallen \\ martin.kolmar@unisg.ch
}

We thank Stefan Bühler, Subhasish Chowdhury, Dana Sisak, Alan Gelder, Thomas Giebe, Radosveta Ivanova-Stenzel, Georgios Katsenos, Kai A. Konrad, Matthias Kräkel, Dan Kovenock, Wolfgang Leininger, Catherine Roux, Marco Runkel, Christian Rusche, and the participants of the Brown Bag Seminars in St. Gallen and Berlin, the Young Researchers' Workshop on Contests and Tournaments in Dortmund and the Workshop on Political Economy at the CESifo in Dresden for insightful comments and helpful suggestions. 


\section{Introduction}

The assumption of selfish payoff-maximizing behavior has long been the basic paradigm in the theory of contests and tournaments, starting with the seminal contributions by Tullock (1980) and Lazear and Rosen (1981). ${ }^{1}$ Evidence from empirical tests of contest theory and behavioral economics in general, however, suggests that people consistently behave as if they were motivated by more general, other-regarding preferences, and the conjecture that social comparisons may influence behavior in contest-like situations has intuitive appeal and shows up in experiments. ${ }^{2}$

Internal promotion tournaments or more generally some form of relative-performance incentive scheme to motivate employees within a firm (Lazear and Rosen (1981)) are a good example: In a number of cases, the employees competing with each other work closely together and have a plethora of opportunities to establish social ties and to evaluate their outcome relative to their peers (see for example Loewenstein, Thompson, and Bazerman (1989); Kräkel (2008); Chen, Ham, and Lim (2011); Altmann, Falk, and Wibral (2012)). Similarly, contests can also be used to analyze litigation and more generally fights (see Baye, Kovenock, and de Vries (2005) and Corchón (2007)). In some legal fields like divorce law, it might also be the case that subjects evaluate outcomes relative to their (former) spouses and that different gender-specific motives play an important role (Wilkinson-Ryan and Small (2008)). And even in territorial conflicts and warfare, the people in charge are often motivated by feelings of national pride and relative national status (see Wallace (1971), and Watson (2008) for an analysis of World War 1). ${ }^{3}$

Consequently, a strand of the contest and tournament literature has emerged that takes distributional preferences (DP) into consideration. ${ }^{4}$ Demougin and Fluet (2003), Grund and Sliwka (2005), Herrmann and Orzen (2008), Fonseca (2009), and Lim (2010) assume probabilistic winnertakes-all contests where players are inequality averse (in the sense of Fehr and Schmidt (1999)) with respect to the final, ex-post distribution of gross or net prizes, and Gill and Stone (2010) develop a model of desert where individuals infer their perception of legitimate claims from their relative investment in effort. Hoffmann and Kolmar (2013) analyze contest behavior if individuals have intention-based fairness preferences in the sense of Rabin (1993).

The present paper contributes to the literature on Fehr and Schmidt (1999) preferences in contests. The novelty of our approach comes in two parts. (i) Not all conflicts have a winner-takes all structure but are rather fights for larger or smaller shares of the prize. Our analysis covers the case of share contests, and the results of our paper are consistent with the empirical findings on

\footnotetext{
${ }^{1}$ See Konrad (2009) for a comprehensive survey of this literature.

${ }^{2}$ See Sheremeta and $\mathrm{Wu}(2012)$ who argue in favor of preferences that are non-separable between consumption and effort and entail pro- and anti-social components.

${ }^{3}$ It is worth noting that along these lines a strand of literature has developed that analyzes the optimal structure of contests, given that players care about their relative position (see Moldovanu, Sela, and Shi (2007), Besley and Ghatak (2008), Dubey and Geanakoplos (2010)).

${ }^{4}$ We use the term distributional preferences as the generic term for the class of preferences that are sensitive to the distribution of prizes or the winning probability.
} 
this type of contest (to be discussed below). A distinction between share and probabilistic contests may appear superfluous because in the standard case of selfish and risk-neutral preferences the difference is exclusively one of interpretation but not of mathematical structure. However, this is no longer the case with distributional preferences. (ii) The formal similarity can be re-established if it is assumed that the distributional concerns of the players in a winner-takes-all contest are not focused on the ex-post but rather on the ex-ante expected distribution of the prize, i.e. winning probability times prize. This is the assumption that we will adopt throughout this paper. We do this for two reasons. First, the explanatory power of a model where individuals are concerned about expected prizes exceeds the explanatory power of alternative models where individuals care for the ex-post prize or have preferences of the Gill and Stone (2010) type. Second, the assumption of ex-ante distributional preferences is consistent with numerous other findings in the literature on $D P$. We will elaborate on these points throughout this introduction.

There are many empirical tests of the two-player probabilistic Tullock contest. Most of them use a simple two-player contest with identical players. These studies have consistently revealed that individuals deviate from the predictions of the selfish Nash equilibrium (NE) in contests and tournaments. See Dechenaux, Kovenock, and Sheremeta (2012) for an excellent survey of contest behavior in experiments. Four patterns emerge. First, subjects often invest more on average than predicted by the selfish Nash equilibrium, which is called overspending. But underspending, i.e. investments in the contest that fall short of the theoretical prediction from the selfish NE, can be observed as well. ${ }^{5}$ Second, if the degree of overspending becomes sufficiently large, aggregate effort exceeds the monetary value of the prize. This effect has been coined overdissipation. Third, individual efforts are distributed over the whole strategy space, with individual behavior being heterogeneous even between stages (overspreading).

Chowdhury, Sheremeta, and Turocy (2012) test contest behavior also for the case of share contests. Their results are qualitatively consistent with the results for probability contests. However, quantitatively, the problems of overspending and overspreading are less pronounced.

The main part of our analysis is devoted to a simultaneous-move two-player contest where individuals may differ with respect to their technological abilities and their distributional preferences. In Section 3 we derive our findings for the case of a Tullock contest-success function. In Appendix $B$ we generalize these findings for the case of a logit-type contest-success function which is frequently used in the literature (see for instance Dixit (1987), Szidarovszky (1997) and Cornes and Hartley (2005, 2012b)). We find the following: (i) If the players are sufficiently similar in abilities and sufficiently inequality averse, there exists a continuum of pure-strategy Nash equilibria (NE) which can be Pareto ranked. In this case the equilibrium strategies are symmetric, although players are allowed to be asymmetric with respect to their abilities as well as preferences. (ii) If inequality aversion is insufficient to overcompensate differences in abilities, the $N E$ is unique. This equilibrium is more egalitarian than the one that emerges with selfish preferences if players are inequality averse.

\footnotetext{
${ }^{5}$ Incidents of underspending are reported by Millner and Pratt (1989, 1991), Davis and Reilly (1998), Potters, Vries, and van Winden (1998), Matros and Armanios (2009), Croson and Önçüler (2005).
} 
If players are inequality prone it may even be less egalitarian than the selfish NE. Interestingly, the less talented player can overcompensate her disadvantage if she cares very strongly for inequality. (iii) Aversion towards disadvantageous inequality $(D I)$ can explain overspending, whereas aversion towards advantageous inequality $(A I)$ can explain underspending. Even though underspending has been observed in experiments, overspending has been observed more frequently. Our results together with the study by Bellemare, Kröger, and van Soest (2008) are able to shed light on this pattern as well. They found that especially well-educated young subjects care almost exclusively about DI. Then, our results imply that the set of underspending equilibria should be very small for this group of subjects. Given that most of the experimental studies have been carried out with students who are well educated and young, our model is able to explain the predominance of overspending. By the same token, disadvantageous inequality aversion can explain overdissipation. (iv) The multiplicity of equilibria with sufficiently homogenous individuals creates an equilibriumselection problem that is difficult to solve even in repeated interactions. Multiple equilibria can therefore explain why individual behavior is so heterogeneous in experiments (overspreading).

In a first extension of the basic model we show that the effect of Fehr and Schmidt (1999) preferences on equilibrium behavior diminishes if the number of players increases. In the limit, as the number of competitors becomes infinitely large, it completely vanishes. In the second extension we analyze sequential investments. Sequential moves lead to the uniqueness of the subgame-perfect NE, and contrary to the findings for selfish players (see Dixit (1987)), a leading favorite may undercommit effort. This effect occurs if the follower has relatively strong advantageous inequality aversion because these preferences can be used as a commitment device for the leading player.

The consistency of the theoretical predictions of our model with the empirical evidence is, however, not sufficient to make it convincing. The burden of proof is twofold. We will first compare the predictive power of our model with the predictive power of the alternative models and second we will argue that our assumption of ex-ante distributional preferences is a plausible and meaningful way to interpret the Fehr and Schmidt (1999) model.

Predictive power of competing theories. There is a large body of literature that tries to clarify the reasons for the above mentioned anomalies. It has evolved broadly along two lines, bounded rationality and DPs; see Baharad and Nitzan (2008) for a survey. ${ }^{6}$

Bounded rationality. Davis and Reilly (1998) conclude that the empirical patterns cannot be explained by risk aversion. See also Cornes and Hartley (2012b) who show theoretically that even as the number of risk-averse participants in a contest increases, the rent will be at most fully dissipated. Potters, Vries, and van Winden (1998) explain the anomalies as a result of randomization of the players over the set of possibly reasonable bids. With respect to overdissipation, Baharad

\footnotetext{
${ }^{6}$ Sheremeta and $\mathrm{Wu}(2012)$ show that non-separable utility between prize and effort together with selfish preferences can explain other behavioral anomalies. They found that in a two prize-spread setting where the contest designer plays an active role, increasing the loser's prize has no impact on effort. Furthermore, the behavior of the contest designer can better be explained with the assumption of non-separable utility. It is not the purpose of our paper to explain these anomalies. We rather join Sheremeta and Wu's conclusion that social preferences should be seen as a complementary explaining variable and explore the former direction.
} 
and Nitzan (2008) focus on distorted perceptions of probabilities. Anderson, Goeree, and Holt (1998) develop a model of bounded rationality that is able to explain the empirical patterns for an all-pay auction. Lim, Matros, and Turocy (2012), and Sheremeta (2011) point to noise and error. Lugovskyy, Puzzello, and Tucker (2010) conclude that the experimental evidence can only be explained by non-monetary incentives of the players, but they do not provide a theoretical model. In the same spirit, Sheremeta (2011) suggests a non-monetary utility of the act of winning. By and large, these different theories are able to explain all types of anomalies, but none of them in a single, unified contest model.

Distributional preferences. Among the literature that focusses on $D P$ in contests and tournaments, the following papers offer a theoretical framework which explicitly models $D P{ }^{7}$ Grund and Sliwka (2005) analyze the outcome of a two-player symmetric promotion tournament where players are inequality averse, and derive the optimal design of this tournament. Herrmann and Orzen (2008) build a model of a symmetric, two-player contest, where competitors are assumed to be spiteful: Players are averse to DI but appreciate AI. They show under which conditions overdissipation is expected to emerge. Fonseca (2009) analyzes the behavior in simultaneous and sequential move two-player contests where players are allowed to be asymmetric with respect to their abilities but symmetric with respect to their inequality aversion. Lim (2010) looks at the optimal share of looser and winner prizes in a motivational tournament framework that allows for social comparison. All these aforementioned papers focus on probabilistic contests in which agents care about the fairness of the ex-post allocation of the prize or the net payoffs. The main insight of these models is that these types of contests can be analyzed like a standard contest where players have selfish preferences and individualized prizes that are recalibrated according to their $D P \mathrm{~s}$. The reason is that $D P \mathrm{~s}$ lead to biased perceptions of the prize, and that the net bias depends on the relative strength of $A I$ and $D I$ aversion or appreciation. If individuals are relatively more concerned about $D I$ $(A I)$, the prize perception is distorted upwards (downwards). Consequently, if players experience for example $D I$ as well as $A I$ aversion of the same magnitude, equilibrium strategies do not deviate from the selfish $N E$ behavior, although final utilities differ substantially (see, for instance, Herrmann and Orzen (2008)). Gill and Stone (2010) develop a model of desert in tournaments where individuals perceive that higher expected payoffs are legitimized by higher effort. If desert concerns are sufficiently strong, asymmetric equilibria exist in which one player works harder and the other slacks off. This is an interesting observation, but does not closely relate to the empirical patterns discussed above.

$\boldsymbol{E x}$-ante inequality aversion. In a probabilistic contest it is a priori not clear whether individuals care for the distribution of the ex-post allocation of the payoff or for the ex ante (expected) payoff,

\footnotetext{
${ }^{7}$ There is a large body of literature which empirically analyzes the effect of $D P$, in particular inequity-aversion on players behavior (see Balafoutas, Kerschbamer, and Sutter (2012), Bartling, Fehr, Maréchal, and Schunk (2009), Eisenkopf and Teyssier $(2009,2010)$.)
} 
and the Fehr and Schmidt (1999) model is open for both interpretations. ${ }^{8}$

Applying distributional preferences to the ex post distribution of income is a form of egalitarianism that focusses exclusively on outcomes. In a Meta study, Konow (2003) argues that the majority of studies that test whether individuals care for the equality of outcomes in this sense are unfavorable to the descriptive value of this form of egalitarianism. Equality of outcomes is only seen as fair in the absence of any other variable individuals consider relevant for justice. This hypothesis is consistent with Brock, Lange, and Ozbay (2010) who show that with risky outcomes in dictator games, the assumption that individuals compare outcomes exclusively on an ex post or ex ante basis cannot explain behavior. Moreover, there is strong support for the hypothesis that individuals take actions into consideration when evaluating the distribution of an allocation (Konow (2003)); people seem to ask if individuals deserve the outcome of some transaction based on individual characteristics (like ability), actions (like effort), as well as on characteristics of the environment (like the causal relationship between actions and outcomes).

Konow (2003) argues that individuals distinguish between different variables that are potentially relevant to justice on the basis of attribution theory, which implies that people evaluate the extent to which an agent has contributed to the outcome of the interaction (see also Sebald (2010)). Individuals are held accountable for only those variables that they can influence and that contribute to the outcome. This concept of accountability discharges into a responsibility-based equity rule (Farwell and Weiner (1996)) and is consistent with a broad range of empirical findings (see Konow (2003)).

Empirical evidence that both, distributional factors and responsibility matter for players' behavior has first been given by Blount (1995). She shows for an ultimatum game that rejection rates are low if the proposal is randomly proposed but that they go up if the randomization is biased in favor of the proposer. Hence, individuals do not care for ex post inequality but rather for ex ante responsibility. This view is also consistent with the findings of Charness and Levine (2003), who show that the perceived intentions of an opponent, as well as the final outcome matter from the point of view of each player, especially if the outcome is sensitive to chance. ${ }^{9}$ Thus, the current consensus suggests that the simple consequentialist model of social preferences is too simplistic to consistently explain behavior. Furthermore, the strong evidence in favor of responsibility-based preferences support the view that individual preferences rather depend on expected instead of actual outcomes in a contest environment because probabilities are a signal for the actions of

\footnotetext{
${ }^{8}$ Note that the ex-ante interpretation put forward in this paper is very much in the spirit of the original article where the authors claim that the "[...] preference parameters are compatible with the interpretation of intentionsdriven reciprocity" (Fehr and Schmidt, 1999, p. 852). The relationship between ex-ante inequality aversion and intentions-driven reciprocity will become clear throughout the paper.

${ }^{9}$ See also Charness and Rabin (2002), Falk and Fischbacher (2006), Kagel, Kim, and Moser (1996) and Offerman (2002). In the same spirit, Krawczyk (2011) shows that a behavioral model where individuals are interested in the expected (that can be influenced by their actions) and not in the actual outcome (that is also determined by chance moves) is a good predictor for behavior in bargaining games. van't Wout, Kahn, Sanfey, and Aleman (2006) have shown that identical offers are much more frequently rejected in an ultimatum game if the offer was made by a human than was generated by a computer. This finding shows that individuals do not necessarily care about inequality as such, but about inequality willfully induced by other individuals.
} 
the players. ${ }^{10}$ We conclude that individuals may be willing to accept ex-post inequality that is unavoidable given the setup (like, for example, in promotion tournaments), but are very sensitive regarding the chances of winning (being promoted).

The model by Gill and Stone (2010) also exemplifies preferences that follow a responsibility-based equity rule, but with a different focus. Our favorite reading of the different approaches is that the specific type of preferences that becomes the driver of individual behavior ultimately depends on the social context in which the contest is embedded. ${ }^{11}$ Under the controlled conditions in the lab, context is usually eradicated to the largest extend possible, and we can therefore only guess how subjects fill in the blanks. However, given that the predictions of our model are consistent with the evidence from the lab, it is a non-falsified candidate for the explanation of behavior in contests.

The paper proceeds as follows. We introduce the model in Section 2. Section 3 focusses on the two-player case with simultaneous moves. Section 4 extends the analysis to the $n$-player case and analyzes equilibria in a sequential move game. Section 5 concludes. Appendix A collects the more involved proofs, and Appendix $B$ offers a generalization of our findings.

\section{The model}

Consider a contest in which each of two players exerts effort $x_{i} \in \mathbb{R}_{+}$in order to win a prize of common value $V>0$, with $i=\{1,2\} .{ }^{12}$ The share of the prize (or the probability of winning) for player $i$ is determined by a contest success function (CSF), $p_{i}: x_{i} \times x_{j} \rightarrow[0,1]$. In particular, we assume that the CSF is given by a logit form CSF in which each player's impact of effort in the contest is represented by an impact function $f: \mathbb{R}_{+} \rightarrow \mathbb{R}_{+}$that is continuous, twice differentiable, increasing and concave.

\section{Assumption 1 (Contest success function)}

The CSF is given by

$$
p_{i}(\mathbf{x})= \begin{cases}\frac{f_{i}\left(x_{i}\right)}{f_{i}\left(x_{i}\right)+f_{j}\left(x_{j}\right)} & \text { for } \mathbf{x} \neq 0, \\ \frac{1}{2} & \text { for } \mathbf{x}=0,\end{cases}
$$

with $\mathbf{x}=\left(x_{i}, x_{j}\right), f_{i}(0)=0$ and $f_{i}^{\prime}\left(x_{i}\right)>0 \geq f_{i}{ }^{\prime \prime}\left(x_{i}\right)$, for $i, j=\{1,2\}$ and $i \neq j .{ }^{13}$

\footnotetext{
${ }^{10}$ In this respect, the utility structure in our model is equivalent to the one used in the rank-order tournament model of Baye, Kovenock, and de Vries (2012), who analyze equilibrium behavior in a two-player complete-information all-pay auctions. In one of the applications players are assumed to be inequality averse with respect to the effort invested.

${ }^{11}$ See Kahneman and Miller (1986) for the seminal contribution on the context-dependency of justice.

${ }^{12}$ The asymmetry between contestants that will be introduced below implies that the assumption of equal valuations is not restrictive for the structure of the equilibria, see Siegel (2009). The results on overspending and overdissipation depend quantitatively but not qualitatively on this convention.

${ }^{13}$ This general CSF has been frequently used (see, for instance, Dixit (1987), Szidarovszky (1997), Cornes and Hartley $(2005,2012 b))$. Assumption (1) is for example fulfilled by the following CSF:
}

$$
p_{i}(\mathbf{x})= \begin{cases}\frac{\eta_{i} x_{i}^{r}}{\eta_{i} x_{i}^{r}+\eta_{j} x_{j}^{r}}, & \text { for } \mathbf{x} \neq 0, \\ \frac{1}{2}, & \text { for } \mathbf{x}=0 .\end{cases}
$$


Thus, player $i$ 's prize share or expected prize $z_{i} \equiv p_{i} V$ is monotonically increasing (decreasing) in $i$ 's (j's) effort.

We assume that players do not only care about their (expected) material payoff $\left(z_{i}\right)$ and effort investment $\left(x_{i}\right)$, but also about the distribution of the (expected) material payoffs. To capture this concern we use (slightly modified) Fehr and Schmidt (1999) fairness preferences.

\section{Assumption 2 (Preferences)}

Given $a_{i} \in \mathbb{R}_{+}, b_{i} \in \mathbb{R}$, and $a_{i} \geq\left|b_{i}\right|$, individual preferences can be described by the following utility function:

$$
\pi_{i}\left(\mathbf{z}, x_{i}\right)=z_{i}-a_{i} \max \left\{-\Delta z_{i}, 0\right\}-b_{i} \max \left\{\Delta z_{i}, 0\right\}-x_{i},
$$

with $\mathbf{z}=\left(z_{1}, z_{2}\right)$, and $\Delta z_{i} \equiv z_{i}-z_{j}$, for $i, j=\{1,2\}$ and $i \neq j$.

The second term represents the disutility from disadvantageous inequality $(D I)$ which depends on the non-negative parameter $a_{i} . \quad a_{i} \geq 0$ rules out that $i$ enjoys DI. The third term represents the (dis)utility from advantageous inequality $(A I)$. Player $i$ is averse to $A I$ if $b_{i}>0$ and prone to $A I$ if $b_{i}<0 .\left|b_{i}\right| \leq a_{i}$ implies that players are more concerned about $D I$ than about $A I^{14}$

Two remarks are in order. First, like Herrmann and Orzen (2008) and Fonseca (2009) (but different to Grund and Sliwka (2005) and Lim (2010)) we use the convention that distributional considerations are restricted to the gross (expected) prize, so that effort costs are excluded. The qualitative results of our model do not depend on this convention. Second, distributional concerns refer to $\left\{z_{i}, z_{j}\right\}=\left\{p_{i} V, p_{j} V\right\}$, which is the only consistent formulation for the case of a share, but not for the case of a probabilistic contest. In a probabilistic contest, $\left\{p_{i} V, p_{j} V\right\}$ refers to the ex-ante expected prize. The previous literature on probabilistic contests (Herrmann and Orzen (2008), Fonseca (2009), Grund and Sliwka (2005), and Lim (2010)) used the different convention that players care for the ex-post allocation of the prize $\{V, 0\}$ or $\{0, V\}\left(\left\{V-x_{1},-x_{2}\right\}\right.$ or $\left\{-x_{1}, V-x_{2}\right\}$ for the case of net prizes). ${ }^{15}$

In what follows we will distinguish between the following types of players:

Definition 1 (Type of player)

Given assumption (2) we will distinguish between the following types of players contingent on the value of coefficients $a_{i}$ and $b_{i}$. Player $i$ is

- selfish if $a_{i}=b_{i}=0$,

with $\eta_{i}, \eta_{j} \in \mathbb{R}_{++}$and $r \in(0,1]$. See for instance Tullock (1980), Nitzan (1994), Baharad and Nitzan (2008) for the CSF in (2) with $\eta_{i}=\eta_{j}=1$ (which has been axiomatized by Skaperdas (1996)) and Gradstein (1995), Corchón (2007) and Corchón and Dahm (2010) for the general case with $\eta_{i} \geq \eta_{j}$ (axiomatized by Clark and Riis (1998)).

${ }^{14}$ Given the upper bound on $\left|b_{i}\right|$, players are loss averse in social comparison, so that players value gains less than losses given the competitor's material payoff as a reference point (see for instance Kong (2008) and Cornes and Hartley (2012a) for the literature on loss aversion in contests, where the reference level of material payoff is exogenously given). From a technical standpoint the upper bound on $\left|b_{i}\right|$ assures the continuity of the bestresponse function.

${ }^{15}$ Please refer to the discussion in the introduction. 
- inequality averse if $a_{i}>0, b_{i} \geq 0$,

- inequality prone if $a_{i}>0, b_{i}<0$.

In what follows, it proves to be more convenient to denote by $y_{i}=f_{i}\left(x_{i}\right)$ player $i$ 's impact in the contest and work with an isomorphic problem that uses the inverse function $h_{i}\left(y_{i}\right)=f_{i}^{-1}\left(y_{i}\right)$ $\left(f_{i}\left(x_{i}\right)\right.$ is strictly monotonic, therefore the inverse exists), with $h_{i}^{\prime}\left(y_{i}\right) \geq 0, h_{i}^{\prime}\left(y_{i}\right)>0$ for $y_{i}>0$ and $h_{i}{ }^{\prime \prime}\left(y_{i}\right) \geq 0$ as costs-of-impact function. ${ }^{16}$

Denote $\Delta y_{i}=y_{i}-y_{j}$ and $\mathbf{y}=\left(y_{i}, y_{j}\right)$. The impact-formulation together with assumptions (1) and (2) imply that we can rewrite the utility function as follows:

$$
\pi_{i}(\mathbf{y})=\frac{V}{y_{i}+y_{j}}\left(y_{i}-a_{i} \max \left\{-\Delta y_{i}, 0\right\}-b_{i} \max \left\{\Delta y_{i}, 0\right\}\right)-h_{i}\left(y_{i}\right),
$$

for $\mathbf{y} \neq \mathbf{0}$ and $\pi_{i}(\mathbf{0})=V / 2$ for $\mathbf{y}=\mathbf{0}$. This formulation reveals why ex-ante inequality concerns do not only allow interpreting distributional preferences as an example for a consequentialist fairness norm. It can be given a procedural interpretation by recognizing that impacts $\left\{y_{i}, y_{j}\right\}$ are measures for efforts $\left\{x_{i}, x_{j}\right\}$ that themselves can be seen as measures for the intentions of the two players. Our conventions therefore gives the idea of intentions-driven fairness (Fehr and Schmidt (1999)) a meaningful interpretation in a probabilistic contest framework. ${ }^{17}$

The partial derivative of (4) with respect to $y_{i}$ yields player $i$ 's marginal utility:

$$
\frac{\partial \pi_{i}(\mathbf{y})}{\partial y_{i}}=\left\{\begin{array}{c}
\beta_{i} V \\
V \\
\alpha_{i} V
\end{array}\right\} \times \frac{y_{j}}{\left(y_{i}+y_{j}\right)^{2}}-h_{i}^{\prime}\left(y_{i}\right) \text { for } \Delta y_{i}\left\{\begin{array}{l}
> \\
= \\
<
\end{array}\right\} 0,
$$

with $\alpha_{i}=1+2 a_{i}$ and $\beta_{i}=1-2 b_{i}$. (5) shows that within both inequality regimes the marginal return of $y_{i}$ depends on "perturbed" valuations of the prize $\left\{\beta_{i} V, \alpha_{i} V\right\}$. In a situation of $D I$ $\left(\alpha_{i}>1\right)$ the marginal return of $y_{i}$ exceeds the one for a selfish player, whereas it can exceed or fall short of the one for a selfish player in case of $A I$, depending on whether player $i$ is inequality prone $\left(\beta_{i}<1\right)$ or averse $\left(\beta_{i} \geq 1\right)$.

\section{A two-player simultaneous move game - the linear case}

In this section we present the case of a two-player simultaneous move game in which the assumed CSFs are a proper subset of those introduced in Assumption 1. In particular, we make the assumption of a linear impact function for both players because it allows us to develop the intuitions for the results in a very intuitive manner. Appendix B provides an analysis for the general CSF introduced in assumption (1).

\footnotetext{
${ }^{16}$ Any fixed-prize contest with a logit-form CSF, concave impact function and unit effort costs can be transformed into an equivalent lottery contest with convex costs. Asymmetries between both players with respect to their impacts in the contest can be transformed into asymmetries with respect to their costs-of-impact function (see Cornes and Hartley (2003, 2005) and Corchón (2007)).

${ }^{17}$ Note that this interpretation of intentions differs from the one in Rabin (1993). See Hoffmann and Kolmar (2013) for an analysis of Rabin-type preferences in contests.
} 


\section{Assumption 3}

Suppose that the CSF is given by (1), with $f_{i}\left(x_{i}\right)=x_{i} / c_{i}, c_{i}>0$, so that $h_{i}\left(y_{i}\right)=c_{i} y_{i}$.

Partial differentiating (5) with respect to $y_{i}$ shows that player $i$ 's utility function is strictly concave in $y_{i}$. Thus, setting $\partial \pi_{i}(\mathbf{y}) / \partial y_{i}=0$ leads to the unique best response for every $y_{j}>0$.

\section{Lemma 1}

Under assumptions (2) and (3), player $i$ 's best response function is given by

$$
B R_{i}\left(y_{j}\right)= \begin{cases}\sqrt{\frac{\beta_{i} V y_{j}}{c_{i}}}-y_{j}, & \text { for } y_{j}<y_{j}^{\beta}, \\ y_{j}, & \text { for } y_{j} \in\left[y_{j}^{\beta}, y_{j}^{\alpha}\right], \\ \sqrt{\frac{\alpha_{i} V y_{j}}{c_{i}}}-y_{j}, & \text { for } y_{j} \in\left(y_{j}^{\alpha}, \hat{y}_{j}\right), \\ 0, & \text { for } y_{j} \geq \hat{y}_{j},\end{cases}
$$

with $y_{j}^{\beta} \equiv \frac{\beta_{i} V}{\left(4 c_{i}\right)}, y_{j}^{\alpha} \equiv \frac{\alpha_{i} V}{\left(4 c_{i}\right)}$ and $\hat{y} \equiv \frac{\alpha_{i} V}{c_{i}}$.

\section{Proof.}

Assume that $y_{j}<\hat{y}_{j}$, so that an interior solution to (5) exists. Given that $\beta_{i}$ matters for player $i$ if $\Delta y_{i}>0$, and that $\alpha_{i}$ matters if $\Delta y_{i}<0$, we will henceforth call $\Delta y_{i}>0$ player $i$ 's $\beta$-regime and $\Delta y_{i}<0$ player $i$ 's $\alpha$-regime. The regimes can be interpreted as if player $i$ had two virtual types $\alpha_{i}, \beta_{i}$ with selfish preferences who differ in their valuation of the prize, $\left\{\beta_{i} V, \alpha_{i} V\right\}$. The best responses for these two types would be $B R_{i}^{\alpha}\left(y_{j}\right)=\max \left\{\sqrt{\alpha_{i} V y_{j} / c_{i}}-y_{j}, 0\right\}$ and $B R_{i}^{\beta}\left(y_{j}\right)=$ $\max \left\{\sqrt{\beta_{i} V y_{j} / c_{i}}-y_{j}, 0\right\}$ (cf. eq. (6)). Both best responses reach their maximum at $y_{i}=y_{j}$. Hence,

$$
B R_{i}^{\alpha}\left(y_{j}\right)\{\gtreqless\} y_{j} \Leftrightarrow y_{j}\{\lesseqgtr\} y_{j}^{\alpha} \text { and } B R_{i}^{\beta}\left(y_{j}\right)\{\gtreqless\} y_{j} \Leftrightarrow y_{j}\{\lesseqgtr\} y_{j}^{\beta} .
$$

Both types' best-response functions are presented in Figure 1. The best responses given in (6) can be constructed as follows: From (5) is follows that $y_{i}=y_{j}<y_{j}^{\beta}$ can never be a best response for player $i$ since there is always a profitable upward deviation which is consistent with the $\beta$-regime. At point $\mathcal{A}$ in Figure 1 player $i$ 's utility is equal to $\hat{\pi}_{i}$. The indifference curve representing all the strategy-profiles which yield this utility $\left(\pi(\mathbf{y})=\hat{\pi}_{i}\right)$ is given by the solid curve going through $\mathcal{A}$. It consists of two parts: (1) Given that $y_{i}>y_{j}$ any $\mathbf{y}$ yielding $\hat{\pi}_{i}$ is identical to any $\mathbf{y}$ which lies on the $\beta$-type's indifference curve and yields the same utility. However, this type's indifference curve is no longer relevant for $y_{i}<y_{j}$ (which is represented by the grey dashed curve beneath the 45 -degree line), since the $\alpha$-types indifference curve becomes relevant. (2) This type's indifference curve is thus represented by the thick black (the grey dashed) curve if $y_{i}<y_{j}\left(y_{i}>y_{j}\right)$. Consequently, any upward deviation from $y_{i}=y_{j}$ and towards the $\beta$-type's best response function must lie to the east of that type's (and therefore player $i$ 's) indifference curve, yielding a higher utility.

By the same token, for $y_{j}>y_{j}^{\alpha}, B R_{i}^{\alpha}\left(y_{j}\right)<y_{j}$ such that for any $y_{i}=y_{j}>y_{j}^{\alpha}$ there is always a profitable downward deviation which is consistent with the $\alpha$-regime (see point $\mathcal{C}$ in Figure 1).

For the intermediate cases $y_{j} \in\left[y_{j}^{\beta}, y_{j}^{\alpha}\right]$ we pick some arbitrary pair $y_{i}=y_{j}$ (point $\mathcal{B}$ ) and check for profitable deviations for player $i$. Note that any upward deviation $y_{i}>y_{j}$ brings player $i$ into 
the $\beta$-regime, whereas any downward deviation $y_{i}<y_{j}$ brings player $i$ into the $\alpha$-regime. However, for any $y_{j} \in\left[y_{j}^{\beta}, y_{j}^{\alpha}\right]$ we find that $B R_{i}^{\alpha}>y_{j}>B R_{i}^{\beta}$. Consequently, there is no profitable upward or downward deviation and $B R_{i}\left(y_{j}\right)=y_{j}$ for any $y_{j} \in\left[y_{j}^{\beta}, y_{j}^{\alpha}\right]$. Hence, the best response function of player $i$ is given by (6).

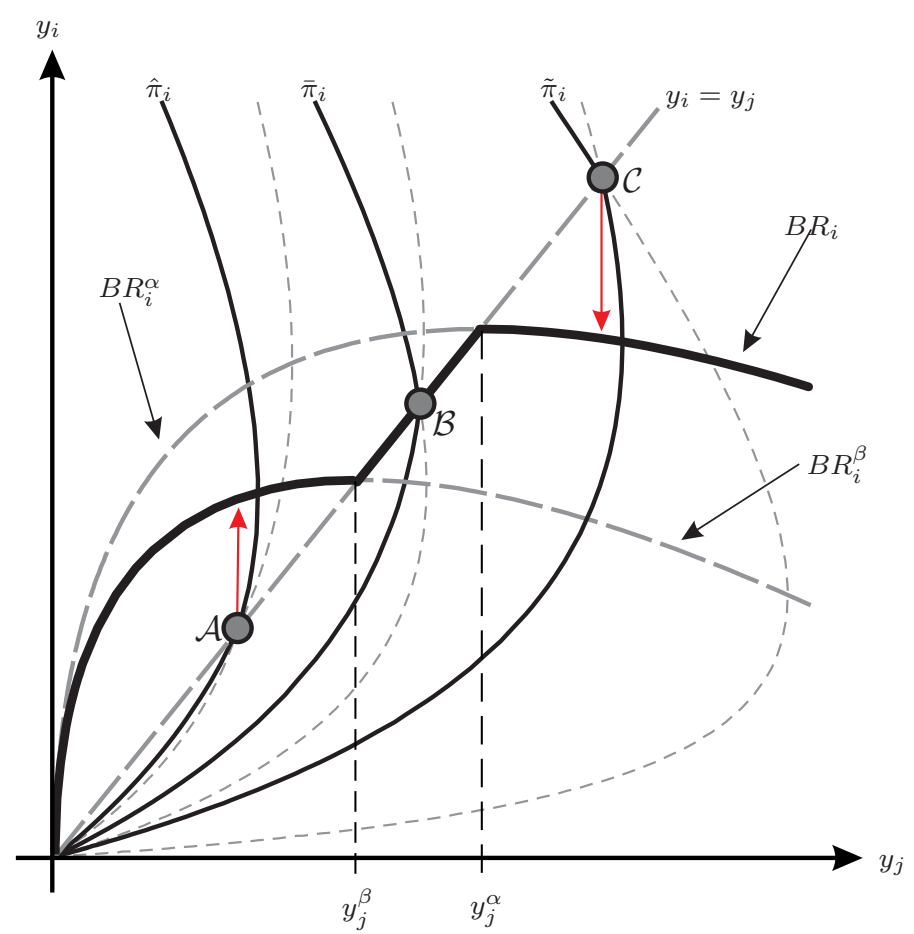

Figure 1

Best response function in the presence of distributional preferences

Apparently, the different prize perceptions create an incentive for player $i$ to deviate from any $y_{i}=y_{j}$ if $y_{j}$ is sufficiently small $\left(y_{j}<y_{j}^{\beta}\right)$. In case of an inequality averse player, the disutility stemming from the resulting $A I$ aversion is then overcompensated by an increase in $p_{i}(\mathbf{y})$. If $y_{j}$ is sufficiently large $\left(y_{j}>y_{j}^{\alpha}\right)$ player $i$ has, again, an incentive to deviate from the egalitarian allocation. He then chooses some $y_{i}<y_{j}$, which is optimal if the resulting disutility stemming from the $D I$ aversion and the decrease in $p_{i}(\mathbf{y})$ is overcompensated by lower effort costs.

We turn to the analysis of the simultaneous-move Nash equilibrium $(N E)$ now. It is a wellestablished result in the literature on contests that, under assumption (3), the $N E$ in case of selfish players $\left(\mathbf{y}^{*}=\left(y_{i}^{*}, y_{j}^{*}\right)\right)$ is given by ${ }^{18}$

$$
y_{i}^{*}=\frac{c_{j} V}{\left(c_{i}+c_{j}\right)^{2}}, \quad y_{j}^{*}=\frac{c_{i} V}{\left(c_{i}+c_{j}\right)^{2}} .
$$

${ }^{18}$ See Konrad (2009). 
In Dixit's (1987) terminology, a selfish player in a two-player contest is a favorite (underdog) if his winning probability at the $N E$ is larger (smaller) than one-half. Given the equilibrium strategies in the case of selfish preferences (cf. eq. (8)) we conclude that player $i$ is the favorite of the game $\left(y_{i}^{*}>y_{j}^{*}\right)$ if and only if $c_{j}>c_{i} .{ }^{19}$

Next we determine the $N E$ for the case of players with DP. It turns out that the cross-player inequality aversion ratio $\xi_{j}^{i} \equiv \beta_{i} / \alpha_{j}$ plays a dominant role. It takes into consideration player $j$ 's $D I$ aversion and player $i$ 's $A I$ aversion or proneness, because these two factors drive behavior in any situation of inequality. The following proposition summarizes the structure of the NE.

\section{Proposition 1 (Simultaneous-move Nash equilibrium)}

Under assumptions (2) and (3) we find the following:

1. There exists a unique and interior Nash equilibrium if and only if one of the cross-player inequality aversion ratios is at least as high as the corresponding marginal costs ratio. More precisely,

$$
\left(\xi_{i}^{j}\right)^{-1} \geq \xi_{j}^{i} \geq \frac{c_{i}}{c_{j}} \Leftrightarrow y_{i}^{N}=\frac{\alpha_{j} \beta_{i}^{2} c_{j} V}{\left(\alpha_{j} c_{i}+\beta_{i} c_{j}\right)^{2}}, y_{j}^{N}=\frac{\alpha_{j}^{2} \beta_{i} c_{i} V}{\left(\alpha_{j} c_{i}+\beta_{i} c_{j}\right)^{2}} .
$$

2. Suppose both players are non-selfish (either inequality averse or prone). Then there exists a continuum of equilibria if and only if both cross-player inequality aversion ratios are smaller than the corresponding marginal costs ratio. More precisely,

$$
\xi_{j}^{i}<\frac{c_{i}}{c_{j}}<\left(\xi_{i}^{j}\right)^{-1} \Leftrightarrow y_{i}^{N}=y_{j}^{N} \in\left[\underline{y}^{N}, \bar{y}^{N}\right],
$$

$$
\text { with } \underline{y}^{N} \equiv \max \left\{y_{i}^{\beta}, y_{j}^{\beta}, 0\right\} \text { and } \bar{y}^{N} \equiv \min \left\{y_{i}^{\alpha}, y_{j}^{\alpha}\right\} \text {. }
$$

Proof. Appendix A.1

We start the discussion of Proposition 1 with the symmetric case $\alpha_{i}=\alpha, \beta_{i}=\beta, c_{i}=c$. In case of selfish preferences $(\alpha=\beta=1)$ the condition in (9) is satisfied with equality, implying that (10) fails to hold. Thus, we have a unique and egalitarian $N E$ with the following equilibrium strategies: $\mathbf{y}^{N}=\left(\frac{V}{4 c}, \frac{V}{4 c}\right)$ that coincide with the selfish $N E\left(\mathbf{y}^{*}\right)$. Denote $\mathcal{N}(\mathbf{y})=\left\{y_{i}=y_{j} \mid \underline{y}^{N} \leq y \leq \bar{y}^{N}\right\}$ the set of egalitarian Nash equilibria. Introducing $D I$ aversion $(\alpha>1)$ leads to a continuum of equilibria (with $\bar{y}^{N}=\frac{\alpha V}{4 c}$ ) since (9) fails to hold. Then $\mathbf{y}^{*} \in \mathcal{N}(\mathbf{y})$ if $\beta \leq 1$ and $\mathbf{y}^{*} \notin \mathcal{N}(\mathbf{y})$ if $\beta>1$. The reason is that $\underline{y}^{N} \leq y_{i}^{*}$ if $\beta \leq 1$ whereas $\underline{y}^{N}>y_{i}^{*}$ if $\beta>1$. This implies that (i) distributional preferences create a coordination problem between players, (ii) $\mathcal{N}(\mathbf{y})=\left\{\mathbf{y}^{*}\right\}$ as $\alpha$ and $\beta$ converge towards one, and (iii) for $\beta \leq 1$ we get $\mathbf{y} \gtreqless \mathbf{y}^{*}$ for all $\mathbf{y} \in \mathcal{N}(\mathbf{y})$, whereas for $\beta>1$ we get $\mathbf{y}>\mathbf{y}^{*}$ for all $\mathbf{y} \in \mathcal{N}(\mathbf{y})$.

The economic intuition for (ii) is obvious but has an interesting implication with respect to the expected under- or overdissipation of prizes compared to the selfish NE: The range of equilibrium deviations from the selfish $N E$ depends on the strength of the $D P$. Suppose $\beta<1$. Then, the

\footnotetext{
${ }^{19}$ Henceforth we will keep the above definition of favorite/underdog for the case of players with DP.
} 
smaller $\beta$, the larger the set of underspending equilibria, and the larger $\alpha$, the larger the set of overspending equilibria. Hence, if equilibrium coordination happens somewhat probabilistically, overspending becomes the more likely the more agents are concerned about $D I$ and the less they are concerned about $A I$. Moreover, if players are $A I$ prone, underspending equilibria never emerge since $\mathbf{y}^{*} \notin \mathcal{N}(\mathbf{y})$ and $\mathbf{y}>\mathbf{y}^{*}$ for all $\mathbf{y} \in \mathcal{N}(\mathbf{y})$.

The intuitions for (i) and (iii) are closely related. Suppose $\alpha>1$ and $\beta<1$ so that players are inequality averse. Then inequality aversion penalizes player $i$ if he becomes the favorite $\left(y_{i}^{N}>y_{j}^{N}\right)$ or if he becomes the underdog $\left(y_{i}^{N}<y_{j}^{N}\right)$. If player $i$ invests, for example, $y_{i}^{*}$, player $j$ would not be willing to deviate from $y_{j}=y_{i}^{*}$ with selfish preferences. With inequality aversion, the utility loss in case of deviation is accentuated because investing more brings the player in the favorite position, and the inequality-aversion factor $\beta$ becomes relevant. Investing less brings him in the underdog position, and the inequality-aversion factor $\alpha$ becomes relevant. Now suppose that player $i$ chooses some $y_{i}=y_{i}^{*}+\varepsilon$, with $\varepsilon \rightarrow 0^{+}$. In case of selfish preferences we find that $B R_{j}\left(y_{i}\right)<y_{i}$, so that a selfish player's utility decreases if he chooses $y_{j}=y_{i}>y_{i}^{*}$. However, this effect is only second order whereas the additional penalty due to player $j$ 's $D I$ aversion is first order. This prevents player $j$ from deviating downwards. By the same token, player $i$ would also not react by investing more because this deviation would be penalized via $i$ 's $A I$ aversion. And a similar reasoning holds for $y_{i}=y_{i}^{*}-\varepsilon$, with $\varepsilon \rightarrow 0^{+}$.

Now suppose $\beta>1$ so that players are inequality prone. Again, we start with $y_{j}=y_{i}^{*}$. Deviating to some $y_{j}=y_{i}^{*}+\varepsilon$ would decrease a selfish player's utility. However, this effect is dominated by the additional utility that stems from the $A I$ resulting from that deviation if players are inequality prone. Thus, $y_{j}=y_{i}^{*}$ cannot be a $N E$. Now suppose $y_{i} \in\left(y_{i}^{\beta}, y_{i}^{\alpha}\right)$. Then we can replicate the above reasoning for the case of inequality-averse players.

Next we will analyze the case of two heterogeneous and non-selfish players with equal marginal costs. Define $a^{\min }=\min \left\{a_{i}, a_{j}\right\}, b^{\min }=\min \left\{b_{i}, b_{j}\right\}$, so that $\bar{\beta}=1-2 b^{\min }$ and $\underline{\alpha}=1+2 a^{\min }$. Then the following corollary follows directly from Proposition (1):

\section{Corollary 1}

Under assumptions (2) and (3), the set of NE-strategies is given by

$$
y_{i}^{N}=y_{j}^{N} \in\left[\max \left\{0, \frac{\bar{\beta} V}{4 c}\right\}, \frac{\underline{\alpha} V}{4 c}\right]
$$

for $c_{i}=c_{j}=c$ and $\xi_{j}^{i}<\frac{c_{i}}{c_{j}}<\left(\xi_{i}^{j}\right)^{-1}$.

\section{Proof. Immediate.}

The difference to the symmetric case discussed before is that the smaller of the two coefficients $b_{i}$ and $b_{j}$ determines the lower bound of the continuum (cf. eq. (10)) as long as $b^{\text {min }} \leq 1 / 2$. Moreover, the smaller of the two coefficients $a_{i}$ and $a_{j}$ determines the upper bound of the continuum. Corollary (1) thus reveals that compared to the homogenous case, heterogeneous distributional preferences never expand the equilibrium set. Interestingly, $\bar{y}^{N}\left(\underline{y}^{N}\right)$ is determined by player $i$ 
if $\underline{\alpha}=\alpha_{i}\left(\bar{\beta}=\beta_{i}\right)$. Thus, the upper bound (lower bound) of $\mathcal{N}(\mathbf{y})$ is determined by the player with the weakest $D I$ aversion (weakest $A I$ aversion or strongest $A I$ proneness). These findings reveal that one-sided distributional preferences will (will not) influence equilibrium behavior if a selfish player encounters an inequality prone (inequality averse) player since it is determined exclusively by the inequality prone (selfish player). This result is in stark contrast to the one for the sequential-move game that we will discuss below.

See Figure 2 for an example of a set of egalitarian solutions. The dashed graphs are the bestresponse functions for the case of selfish preferences, and their intersection $\left(\mathbf{y}^{*}\right)$ represents the selfish NE. Since $y_{i}^{N}>y_{j}^{N}$, player $j$ is the underdog and player $i$ is the favorite at $\mathbf{y}^{*}$. The

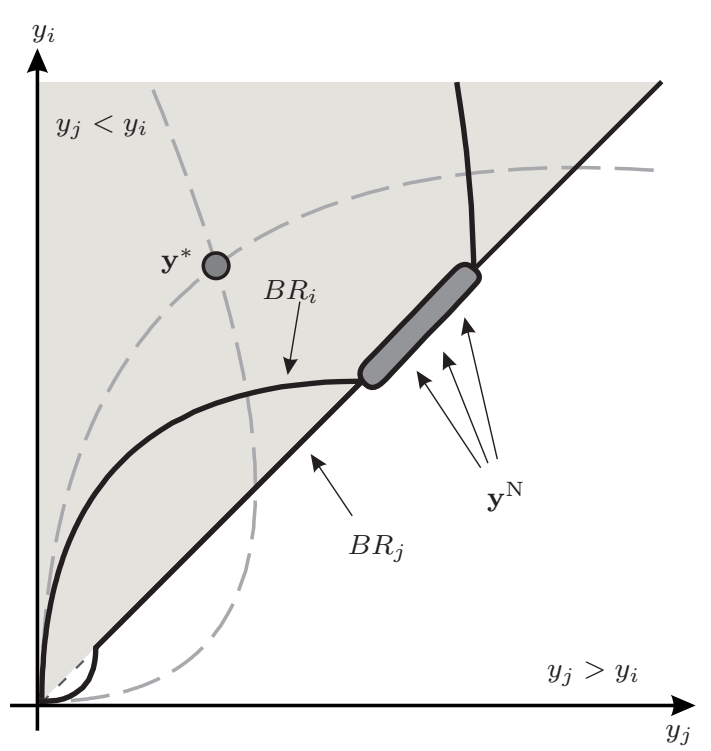

Figure 2

A continuum of $\mathrm{NE}$

in case of two inequality averse players

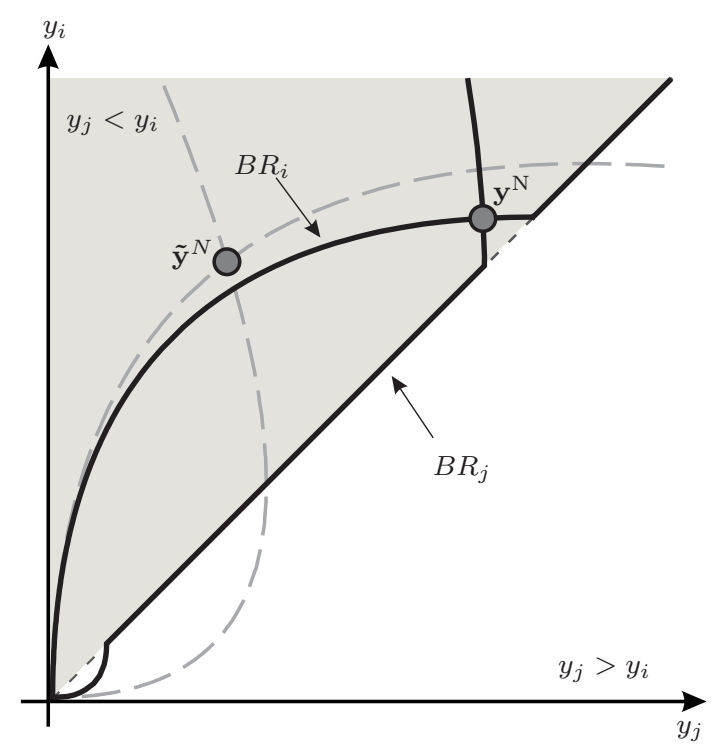

Figure 3

A unique NE

in case of two inequality averse players

solid lines are the best-response functions for the case of non-selfish preferences. Apparently, both players are inequality averse since their $\beta$-type's best-response functions (the part of each player's best response function that is larger than the corresponding competitor's strategy) lie below the best-response functions for selfish preferences. Given inequality aversion, player $i$ becomes less aggressive and player $j$ more aggressive in the relevant range $\left(y_{i}>y_{j}\right)$ as we move from $\mathbf{y}^{*}$ towards $\mathbf{y}^{N}$. Evidently, $D P$ are sufficiently strong to push the $N E$ on to the 45-degree line. Even more, the $N E$ is no longer unique and the equilibrium set is the whole highlighted segment of the 45-degree line.

Next, we turn to the case of a unique NE. We find the following.

\section{Corollary 2}

If $\left(\xi_{i}^{j}\right)^{-1} \geq \xi_{j}^{i} \geq \frac{c_{i}}{c_{j}}$ then player $i$ 's equilibrium share is never smaller than that of his competitor:

$$
\left(\xi_{i}^{j}\right)^{-1} \geq \xi_{j}^{i}\left\{\begin{array}{l}
> \\
=
\end{array}\right\} \frac{c_{i}}{c_{j}} \Leftrightarrow y_{i}^{N}\left\{\begin{array}{l}
> \\
=
\end{array}\right\} y_{j}^{N} .
$$


If player $i$ 's $\mathrm{AI}$ proneness is stronger than his competitor's DI aversion $\left(\beta_{i}>\alpha_{j} \geq 1\right)$, player $i$ will even end up with a larger share compared to the selfish $\mathrm{NE}\left(y_{i}^{N}>y_{i}^{*}\right)$.

Proof. Immediate.

Corollary (2) shows that whatever the value of $c_{i} / c_{j}$, player $i$ receives a larger (the same) share than (as) his competitor in case $\xi_{j}^{i}>c_{i} / c_{j}\left(\xi_{j}^{i}=c_{i} / c_{j}\right)$. Hence, if $\xi_{j}^{i}>c_{i} / c_{j}, A I$ aversion or proneness matters for player $i$, whereas $D I$ aversion matters for player $j$. Furthermore, if $\beta_{i}>\alpha_{j}$ the $N E$ share of player $i$ will be larger than in the selfish case. For example, if player $i$ is more efficient $\left(c_{j}>c_{i}\right)$ the equilibrium share at $\mathbf{y}^{N}$ is less balanced than at $\mathbf{y}^{*}$, so that $p_{i}\left(\mathbf{y}^{N}\right)>p_{i}\left(\mathbf{y}^{*}\right)>\frac{1}{2}$. However, if player $i$ is less efficient $\left(c_{j}<c_{i}\right)$ we find that he receives a larger share at $\mathbf{y}^{N}$ compared

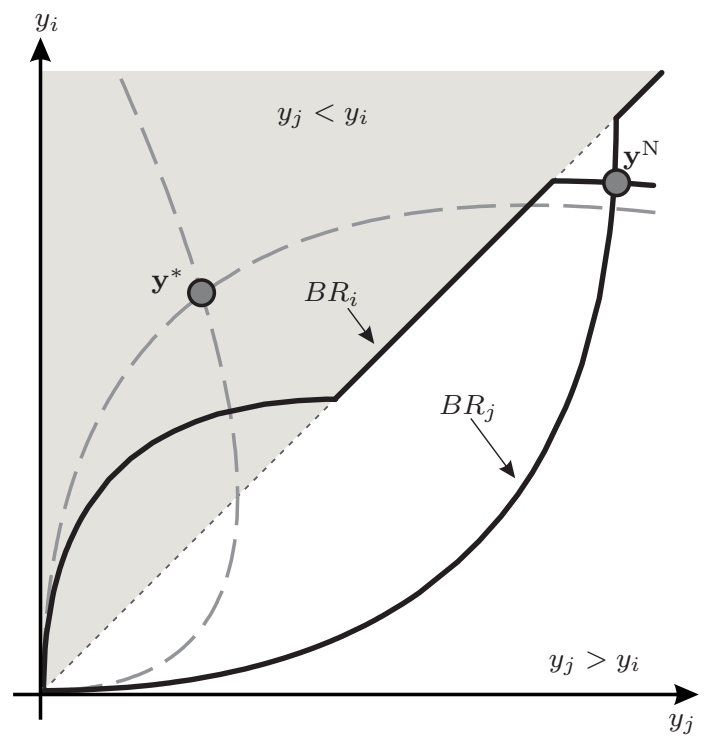

Figure 4

A unique $\mathrm{NE}$

in case player $j$ is inequality prone

to $\mathbf{y}^{*}$, so that $p_{i}\left(\mathbf{y}^{N}\right)>\frac{1}{2}>p_{i}\left(\mathbf{y}^{*}\right)$. This shows that introducing DP can lead to (i) an allocation that is even more unequal than the selfish NE, or (ii) to an allocation that reverses the relative equilibrium share of players' compared to the selfish NE.

See Figure 3 for an example of a unique $N E$ where both players are inequality averse. It has the same structure as Figure 2 with the only exception that players' inequality aversion is not strong enough to completely equalize the outcome. However, the equilibrium strategy profile moves closer to the 45-degree line, showing that $D P$ have a tendency to equalize the outcome in this example. Figure 4 shows the case in which player $i(j)$ is inequality averse (prone), since players $i$ 's ( $j$ 's) $\beta$-type best response functions lies below (above) above his selfish best responses. Here, $y_{j}^{N}>y_{i}^{N}$ so that the less efficient player receives a larger share of the prize. Again, the reason is player $j$ 's distinctive $A I$ proneness. A lack of talent can be overcompensated by strong inequality proneness. 
Note that the properties of a $N E$ depend crucially on the relationship between the inequalityaversion ratio $\left(\xi_{j}^{i}\right)$ and the corresponding marginal-costs ratio $\left(c_{i} / c_{j}\right)$. The farer away $c_{i} / c_{j}$ is from unity, the farer away from the egalitarian allocation $\left(y_{i}=y_{j}\right)$ is the selfish $N E$. In order to compensate for a large technological advantage of player $i\left(c_{j} \gg c_{i}\right), \xi_{j}^{i}$ must be sufficiently small. This requirement is sufficient to reach an egalitarian allocation if $j$ is inequality averse $\left(\beta_{j} \geq 1\right)$. Otherwise, player $j$ 's $A I$ proneness must not be too large compared to $i$ 's $D I$ aversion. If this is the case, distributional considerations perfectly correct the unequal distribution of the prize (in the sense of inducing an egalitarian distribution) that would result from the technological comparative advantage of player $i$.

It is interesting to understand, how the equilibrium changes if the explanatory variables change. The comparative-static analysis can be carried out for the case of a unique equilibrium. In order to do so, it is useful to analyze the players' strategic incentives at $\mathbf{y}^{N}$ if $\xi_{j}^{i} \geq c_{i} / c_{j}$ so that $p_{i}\left(\mathbf{y}^{N}\right) \geq$ $1 / 2 .{ }^{20}$ It is a well-known fact that in the case of two-player contests with selfish preferences the favorite regards strategies as strategic complements $(S C)$ while the underdog regards strategies as strategic substitutes $(S S)$ at the NE. If the share of the prize at the $N E$ is $1 / 2$, both players regard strategies as strategically independent $(S I) .{ }^{21}$ This fact remains true even if players have $\mathrm{DP}$, since

$$
\frac{\partial^{2} \pi_{i}(\mathbf{y})}{\partial y_{i} \partial y_{j}}=\left\{\begin{array}{c}
\beta_{i} V \\
V \\
\alpha_{i} V
\end{array}\right\} \times \frac{\Delta y_{i}}{\left(y_{i}+y_{j}\right)^{3}} \text { for } \Delta y_{i}\left\{\begin{array}{l}
> \\
= \\
<
\end{array}\right\} 0 .
$$

Turning to the comparative statics, we find the following:

$$
\begin{aligned}
& \frac{\partial y_{i}^{N}}{\partial b_{i}}<0, \quad \frac{\partial y_{j}^{N}}{\partial b_{i}} \geq 0 \quad \text { and } \quad \frac{\partial Y^{N}}{\partial b_{i}}<0, \\
& \frac{\partial y_{i}^{N}}{\partial a_{j}} \geq 0, \quad \frac{\partial y_{j}^{N}}{\partial a_{j}}>0 \quad \text { and } \quad \frac{\partial Y^{N}}{\partial a_{j}}>0,
\end{aligned}
$$

with $Y^{N}=y_{i}^{N}+y_{j}^{N}$. A marginal increase in the $A I$ aversion (synonymously: a marginal decrease in the $A I$ proneness) of player $i\left(b_{i}\right)$ decreases the marginal return of $y_{i}$ (cf. eq. (5)) and thus decreases the best response of $i$ for any $y_{j}>0$. For $\xi_{j}^{i}>c_{i} / c_{j}\left(\xi_{j}^{i}=c_{i} / c_{j}\right)$, player $j$ regards strategies as $S S(S I)$ and therefore $y_{j}^{N}$ increases (remains the same). The net effect of an increase in $b_{i}$ on $Y^{N}$ is negative.

An increase in $D I$ aversion of player $j\left(a_{j}\right)$ increases the marginal return of $y_{j}$ (cf. eq. (5)) and increases $j$ 's best response for any $y_{i}>0$. For $\xi_{j}^{i}>c_{i} / c_{j}\left(\xi_{j}^{i}=c_{i} / c_{j}\right)$ player $i$ regards strategies as $S C(S I)$ and therefore $y_{i}^{N}$ increases (remains the same). The net effect of an increase in $a_{j}$ on $Y^{N}$ is positive.

\footnotetext{
${ }^{20}$ The complementary case can be analyzed analogously.

${ }^{21}$ These facts have first been established by Dixit (1987) and have been extensively used, particularly in the literature on endogenous timing in contest. See for instance Baik and Shogren (1992), Leininger (1993), and Hoffmann and Grégoire Rota-Graziosi (2012).
} 
Next, we turn to the issue of overspending and overdissipation in the case of a unique NE. We define overspending as a situation where aggregate effort at $\mathbf{y}^{N}$ is larger than at $\mathbf{y}^{*}$, and overdissipation as a situation where aggregate effort at $\mathbf{y}^{N}$ exceeds the value of the prize $V{ }^{22}$ In order to do so we need to determine the aggregate effort $\left(X \in \mathbb{R}_{+}\right)$at $\mathbf{y}^{N}$ and $\mathbf{y}^{*}$, which can be derived by recognizing that

$$
X=\sum_{i=1}^{2} h_{i}\left(y_{i}\right)
$$

The following results show that distributional preferences can explain rational overdissipation. ${ }^{23}$

Proposition 2.A (Overspending and overdissipation in case of a unique NE)

Under Assumptions (2) and (3) we find that for $\left(\xi_{i}^{j}\right)^{-1} \geq \xi_{j}^{i} \geq \frac{c_{i}}{c_{j}}$ the following holds

$$
X^{N}>X^{*} \Leftrightarrow \frac{\alpha_{j} \beta_{i}\left(\alpha_{j}+\beta_{i}\right)}{\left(\alpha_{j} c_{i}+\beta_{i} c_{j}\right)^{2}}>\frac{2}{\left(c_{i}+c_{j}\right)^{2}}
$$

and

$$
X^{N}>V \Leftrightarrow\left(\beta_{i}+\alpha_{j}-2\right)>\xi_{j}^{i} \frac{c_{j}}{c_{i}}+\left(\xi_{j}^{i} \frac{c_{j}}{c_{i}}\right)^{-1}
$$

Proof. Appendix A.2.

Thus, overspending $\left(X^{N}>X^{*}\right)$ as well as overdissipation $\left(X^{N}>V\right)$ can be explained by means of $D P$ in case of a unique NE. Apparently, a sufficient condition for overspending to emerge is that the favorite player $i$ is less efficient $\left(c_{i}>c_{j}\right)$ so that $\beta_{i}>1$ (cf. Corollary 2). Furthermore, the finding of Cornes and Hartley (2005) that overdissipation is the more likely the more equal the winning probabilities are at the NE, can be pushed beyond selfish preferences. First notice that in the selfish case equal winning probabilities at $\mathbf{y}^{*}$ are an artifact of the players' equal technological abilities. In our model, equal winning probabilities at $\mathbf{y}^{N}$ may even emerge if players are considerable asymmetric with respect to the technological abilities so that $p_{i}\left(\mathbf{y}^{*}\right) \gg \frac{1}{2} \gg p_{j}\left(\mathbf{y}^{*}\right)$. The reason for this is that a huge technological advantage of a player may be overcompensated via the players' DP. Even if a more efficient player $i$ enjoys $A I\left(\beta_{i}>1\right)$ we may find a unique egalitarian NE. Of course, this case only arises if $i$ encounters a sufficiently $D I$ averse player $j$ so that $a_{j}>\left|b_{i}\right|$ and therefore $1>\xi_{j}^{i}=\frac{c_{i}}{c_{j}}$. Second, notice that $y_{i}^{N}=y_{j}^{N}$ at a unique $N E$ if and only if $\xi_{j}^{i}=\frac{c_{i}}{c_{j}}(\mathrm{cf}$. corollary (2)) which is exactly the ratio that minimizes the RHS of (18).

Next, we turn to the case of overspending as well as overdissipation in case of a continuum of equilibria. But before, we will introduce a specific concept of the two above mentioned anomalies that will proof useful in the following proposition.

\footnotetext{
${ }^{22}$ An alternative definition of overdissipation would be that utilities in equilibrium sum to less than zero. Both definitions coincide if the Nash equilibrium is symmetric. They can differ, however, in the asymmetric case because the cognitive costs or gains from inequality would then have to be taken into consideration. Our qualitative findings do not depend on the particular definition of overdissipation used in our model.

${ }^{23}$ The debate about the rationality of overdissipation (Baharad and Nitzan (2008)) makes the point that some form of irrationality is needed to get overdissipation. The reason why overdissipation is not in conflict with full rationality in this model is that deviating players will be plagued by negative emotions because of disadvantageous inequality.
} 


\section{Definition 2 (Probabilistic overspending and overdissipation)}

- Probabilistic overspending emerges if there is a positive probability that the aggregate spending at $\mathbf{y}^{N}$ exceeds the aggregate spending at $\mathbf{y}^{*}$.

- Probabilistic overdissipation emerges if there is a positive probability that aggregate effort at $\mathbf{y}^{N}$ exceeds the value of the prize (see also Baye, Kovenock, and de Vries (1999)).

Proposition 2.B (Overspending and overdissipation in case of a continuum of NE)

Suppose (without loss of generality) that $y_{i}^{\alpha}>y_{j}^{\alpha}$ and $y_{i}^{\beta}<y_{j}^{\beta}$. Under Assumptions (2) and (3) and for $\xi_{j}^{i}<\frac{c_{i}}{c_{j}}<\left(\xi_{i}^{j}\right)^{-1}$ we find

$\left\{\begin{array}{l}\text { probabilistic overspending } \\ \text { overspending }\end{array}\right\}:=\left\{\begin{array}{l}\bar{y}^{N}\left(c_{i}+c_{j}\right) \\ \underline{y}^{N}\left(c_{i}+c_{j}\right)\end{array}\right\}>X^{*} \Leftrightarrow\left\{\begin{array}{c}\alpha_{i} \\ \beta_{i}\end{array}\right\}>\frac{8 c_{i}^{2} c_{j}}{\left(c_{i}+c_{j}\right)^{3}}$,

and

$$
\left\{\begin{array}{l}
\text { probabilistic overdissipation } \\
\text { overdissipation }
\end{array}\right\}:=\left\{\begin{array}{l}
\bar{y}^{N}\left(c_{i}+c_{j}\right) \\
\underline{y}^{N}\left(c_{i}+c_{j}\right)
\end{array}\right\}>V \Leftrightarrow\left\{\begin{array}{c}
\alpha_{i} \\
\beta_{i}
\end{array}\right\}>\frac{4 c_{i}}{c_{i}+c_{j}} .
$$

Proof. Appendix A.3

As before, overspending $\left(X^{N}>X^{*}\right)$ as well as overdissipation $\left(X^{N}>V\right)$ can be explained by means of $D P$ (now for the case of a continuum of NE). Proposition (2.B) implies that a sufficient condition for either of both anomalies to emerge is that the lower bound of the equilibrium set $\left(\underline{y}^{N}\right)$ is large enough. Given $\left\{c_{i}, c_{j}\right\}$, the lower bound uniquely depends on the levels of $\beta_{i}$. Probabilistic overspending and overdissipation however, emerges if the upper bound of the equilibrium set $\left(\bar{y}^{N}\right)$ is sufficiently large. Thus, for given marginal costs $\left\{c_{i}, c_{j}\right\}$, probabilistic overspending and overdissipation emerges if $\alpha_{i}$ is large enough. Note that a larger $\alpha_{i}$ does not influence the equilibrium share of the prize for any player, since it is equal to $p_{i}\left(\mathbf{y}^{N}\right)=p_{j}\left(\mathbf{y}^{N}\right)=\frac{V}{2}$. However, the larger the $\alpha$ of the more selfish player (here player $i$ since $y_{i}^{\alpha}>y_{j}^{\alpha}$ by assumption), the larger the incentive to invest. Since the threshold value in the case of overdissipation exceeds the one in the case of overspending, we find that $\beta_{i}>4 c_{i} /\left(c_{i}+c_{j}\right)$ is a sufficient condition for overspending as well as overdissipation to emerge for all $\mathbf{y} \in \mathcal{N}(\mathbf{y})$.

\section{Extensions}

In this section we discuss two possible extensions of the above model for the case of linear impact functions. In the first subsection we focus attention on contests with more than two players. In the second subsection we analyze a two-player sequential-move game.

\subsection{Extension to $n$ players}

How does competition between players influence the outcome of the game? In order to answer this question we turn to the case of identical $D P$ s. The main result of the analysis is that an increase in 
the number of players reduces the set of equilibria. If the number of players converges to infinity, the set of equilibria converges to the Nash equilibrium with selfish preferences. We postpone a discussion of this finding until after the formal statement of this result.

Let $N$ be the set of players, $c_{i}=1, a_{i}=a \in \mathbb{R}_{+}, b_{i}=b \in \mathbb{R}$ and $|b|<a$, with $i=1, \ldots, n$. In order to have a lean notation denote by $\mathbf{y}=\left\{y_{1}, \ldots, y_{n}\right\}$ a vector of impacts, by $\mathbf{y}_{-i}$ a vector of impacts of all players except $i$, by $Y=\sum_{i} y_{i}$ the sum of impacts, and by $Y_{-i}=\sum_{j \neq i} y_{j}$ the sum of impacts of all players except for player $i$. With these conventions, (4) generalizes to

$$
\pi_{i}\left(y_{i}, \mathbf{y}_{-i}\right)=\frac{y_{i}}{Y} V-\frac{a}{n-1} \sum_{j \neq i} \max \left\{\frac{y_{j}-y_{i}}{Y} V, 0\right\}-\frac{b}{n-1} \sum_{j \neq i} \max \left\{\frac{y_{i}-y_{j}}{Y} V, 0\right\}-y_{i} .
$$

For a given vector of impacts $\mathbf{y}$ denote by $\mathcal{N}^{+}(\mathbf{y})$ and $\mathcal{N}^{-}(\mathbf{y})$ the subsets of players exerting strictly more or less impact than player $i$. Then, (21) can further be simplified to

$$
\pi_{i}\left(y_{i}, \mathbf{y}_{-i}\right)=\frac{y_{i}}{Y} V-\frac{a}{n-1} \sum_{j \in \mathcal{N}^{+}(\mathbf{y})} \frac{y_{j}-y_{i}}{Y} V+\frac{b}{n-1} \sum_{j \in \mathcal{N}^{-}(\mathbf{y})} \frac{y_{j}-y_{i}}{Y} V-y_{i},
$$

and the first-order condition (5) becomes

$$
\frac{\partial \pi_{i}}{\partial y_{i}}=\frac{Y_{-i}}{Y^{2}} V-\frac{a}{n-1} \sum_{j \in \mathcal{N}^{+}(\mathbf{y})} \frac{-Y_{-i}-y_{j}}{Y^{2}} V+\frac{b}{n-1} \sum_{j \in \mathcal{N}^{-}(\mathbf{y})} \frac{-Y_{-i}-y_{j}}{Y^{2}} V-1 .
$$

Note that optimal individual impact in the contest depends only on the aggregate of all other players' impacts, which implies that the above conditions can be used to determine player $i$ 's best response function $B R_{i}\left(y_{1}, \ldots, y_{i-1}, y_{i+1}, \ldots, y_{n}\right)$ as a function of the aggregate of all other players impacts, $B R_{i}\left(y_{1}, \ldots, y_{i-1}, y_{i+1}, \ldots, y_{n}\right)=B_{i}\left(\sum_{j \neq i} y_{j}\right)$. Let us assume that all players except $i$ exert identical impacts $y_{j}$. In this case we can distinguish between two cases, one where all players exert more and one where all players exert less impact than player $i$. In these cases, (23) simplifies to

$$
\frac{\partial \pi_{i}}{\partial y_{i}}=\frac{(1+a) Y_{-i}+a y_{j}}{Y^{2}} V-1=\frac{(1+a)(n-1) y_{j}+a y_{j}}{Y^{2}} V-1
$$

in the former and to

$$
\frac{\partial \pi_{i}}{\partial y_{i}}=\frac{(1-b) Y_{-i}-b y_{j}}{Y^{2}} V-1=\frac{(1-b)(n-1) y_{j}-b y_{j}}{Y^{2}} V-1
$$

in the latter case (we use the convention that $y_{j}=Y_{-i} /(n-1)$ is the generic impact of a player other than $i$ ). The determination of the best response of player $i, B_{i}\left((n-1) y_{j}\right)$, follows the same lines as in the two-player case:

$B_{i}\left((n-1) y_{j}\right)=\left\{\begin{array}{ll}\sqrt{((n-1)-b n) V y_{j}}-(n-1) y_{j}, & y_{j}<\frac{((n-1)-b n) V}{n^{2}} \\ y_{j}, & y_{j} \in\left[\frac{((n-1)-b n) V}{n^{2}}, \frac{((n-1)+a n) V}{n^{2}}\right] \\ \sqrt{((n-1)+a n) V y_{j}}-(n-1) y_{j}, & y_{j} \in\left(\frac{((n-1)+a n) V}{n^{2}}, \frac{((n-1)+a n) V}{(n-1)^{2}}\right] \\ 0, & y_{j}>\frac{((n-1)+a n) V}{(n-1)^{2}}\end{array}\right.$.

It is straightforward to calculate the set of Nash equilibria. The result is summarized in the following proposition. 


\section{Lemma 2 (n-player NE)}

Assume a symmetric $n$-player contest with $c_{i}=1, a_{i}=a, b_{i}=b$ and $a \neq 0 \vee b \neq 0$. For all finite $n$ the game has a continuum of Nash equilibria

$$
y_{i}^{N} \in[\underline{y}, \bar{y}]=\left[\frac{(n-1)-b n}{n^{2}} V, \frac{(n-1)+a n}{n^{2}} V\right] .
$$

Next, we compare this set of Nash equilibria with the symmetric Nash equilibrium in case of selfish preferences. The latter is given by

$$
y_{i}^{*}=\frac{(n-1) V}{n^{2}} .
$$

As for the two-player case, the equilibrium set in case of $D P$ s contains the selfish Nash equilibrium if $b \geq 0$ for all finite $n$, i.e.

$$
\frac{((n-1)-b n) V}{n^{2}}<\frac{(n-1) V}{n^{2}}<\frac{((n-1)+a n) V}{n^{2}} .
$$

More importantly, we can say something about the influence of the number of players on the set of equilibria. This can be done by either comparing $\underline{y}$ with $\bar{y}$ or by comparing $\underline{y}$ and $\bar{y}$ with $y_{i}^{*}$. Both approaches lead to the same conclusion.

\section{Proposition 3 ( $n$-player $N E$ and the number of players)}

Assume a symmetric $n$-player contest with $c_{i}=1, a_{i}=a, b_{i}=b$ and $a \neq 0 \vee b \neq 0$. The equilibrium set is shrinking in $n$ and converges to the Nash equilibrium with selfish preferences if $n$ converges to infinity.

Proof. It is straightforward to calculate $y_{i}^{*}-\underline{y}=b / n, \bar{y}-y_{i}^{*}=a / n$, and $\bar{y}-\underline{y}=(a+b) / n$. All differences are decreasing in $n$, and the set converges to the unique equilibrium $y_{i}^{*}$ for $n \rightarrow \infty$.

This result formally expresses the economic intuition that distributional considerations become less influential in larger groups. In the limit case of atomistic players, even the strongest (but finite) distributional concern is without relevance from a behavioral point of view. The individuals are forced to play the selfish Nash equilibrium because each of them is too small to influence the distribution of the resulting allocation.

\subsection{Sequential-move game}

We now turn to the formal analysis of a sequential-move game, i.e. we allow one of the players to commit to a particular level of effort. The subgame-perfect equilibrium (SPE) of the contest is determined by applying backward induction. Suppose that player $j$ leads. Then, the follower's maximization problem is to determine $y_{i}^{F} \equiv \operatorname{argmax} \pi_{i}(\mathbf{y})$, which yields $y_{i} \equiv B R_{i}\left(y_{j}\right)$. Next, define $\tilde{\pi}_{j}\left(y_{j}\right) \equiv \pi_{j}\left(y_{j}, B R_{i}\left(y_{j}\right)\right)$ as the leader's utility function and $y_{j}^{L} \equiv \underset{y_{j}}{\operatorname{argmax}} \tilde{\pi}_{j}\left(y_{j}\right)$. Under assumptions (2) and (3), $\tilde{\pi}_{j}\left(y_{j}\right)$ takes the following form:

$$
\tilde{\pi}_{j}\left(y_{j}\right)=\frac{V}{y_{j}+B R_{i}\left(y_{j}\right)}\left(y_{j}-a_{j} \max \left\{-\Delta \tilde{y}_{j}, 0\right\}-b_{i} \max \left\{\Delta \widetilde{y}_{j}, 0\right\}\right)-c_{j} y_{j},
$$


with $\Delta \widetilde{y}_{j} \equiv y_{j}-B R_{i}\left(y_{j}\right)$. The leader's FOC is then given by

$$
\frac{d \tilde{\pi}_{j}\left(y_{j}\right)}{d y_{j}}=0 \Leftrightarrow 0=\left\{\begin{array}{l}
\beta_{i} \\
1 \\
\alpha_{i}
\end{array}\right\} \times \frac{B R_{i}\left(y_{j}\right)-B R_{i}^{\prime}\left(y_{j}\right) y_{j}}{\left(y_{j}+B R_{i}\left(y_{j}\right)\right)^{2}} V-c_{j} \text { for } \Delta \widetilde{y}_{i}\left\{\begin{array}{c}
> \\
= \\
<
\end{array}\right\} 0 .
$$

Existence and uniqueness of a solution is ensured by the continuity and strict concavity of the objective function. Note that since the impact elasticity of the best response function $\left(\varepsilon_{i j} \equiv\right.$ $\frac{B R_{i}^{\prime}\left(y_{j}\right) y_{j}}{B R_{i}\left(y_{j}\right)}$ ) is smaller than (equal to) unity for $\Delta \tilde{y}_{j} \neq 0\left(\Delta \tilde{y}_{j}=0\right)$, we find that the numerator of the fraction in (30) is strictly positive (zero). This reflects the fact that as long as $\Delta \tilde{y}_{j}=0$ the equilibrium share of the leader does not change if $y_{j}$ increases marginally, which no longer holds if $\Delta \tilde{y}_{j} \neq 0$.

Solving eq. (30) leads to the following proposition.

\section{Proposition 4 (Subgame perfect equilibrium, SPE)}

Suppose player $j$ leads in a sequential move game. Under assumptions (2) and (3) the SPE $\left(\mathbf{y}^{S_{j}}=\right.$ $\left.\left(y_{j}^{L}, y_{i}^{F}\right)\right)$ has the following properties:

1. If the Stackelberg-leader is an underdog at $\mathbf{y}^{N}$ we find that he undercommits effort compared to the NE:

$$
\left(\xi_{i}^{j}\right)^{-1} \geq \xi_{j}^{i}>\frac{c_{i}}{c_{j}} \Rightarrow y_{j}^{L}<y_{j}^{N} \wedge y_{i}^{F}>y_{i}^{N} .
$$

2. If the Stackelberg-leader is a favorite at $\mathbf{y}^{N}$ we find that

(a) he overcommits effort compared to the $\mathrm{NE}$ if the inequality at $\mathbf{y}^{N}$ is sufficiently large:

$$
\left(\xi_{j}^{i}\right)^{-1} \geq \xi_{i}^{j}>\frac{c_{j}}{c_{i}}\left(1+\sqrt{\frac{\alpha_{i}-\beta_{i}}{\alpha_{i}}}\right) \Rightarrow y_{j}^{L}>y_{j}^{N} \wedge y_{i}^{F}<y_{i}^{N} .
$$

(b) he undercommits effort compared to the $\mathrm{NE}$ if the inequality at $\mathbf{y}^{N}$ is not sufficiently large:

$$
\left(\xi_{j}^{i}\right)^{-1} \geq \xi_{i}^{j} \in\left(\frac{c_{j}}{c_{i}}, \frac{c_{j}}{c_{i}}\left(1+\sqrt{\frac{\alpha_{i}-\beta_{i}}{\alpha_{i}}}\right)\right] \Rightarrow y_{j}^{L}<y_{j}^{N} .
$$

3. If both players have equal winning probability at $\mathbf{y}^{N}$, we find that the Stackelberg-leader will never overcommit effort compared to the NE:

$$
\xi_{i}^{j} \leq \frac{c_{j}}{c_{i}} \leq\left(\xi_{j}^{i}\right)^{-1} \Rightarrow y_{j}^{L}=y_{i}^{F} \leq y_{i}^{N}=y_{j}^{N} .
$$

\section{Proof. See Appendix A.4.}

In case of a leading underdog, the follower regards efforts as SC (cf. eq. (13)). Thus, since an increase in $y_{i}$ always has a negative impact upon $j$ 's payoff $\left(\partial \pi_{j}(\mathbf{y}) / \partial y_{i}<0\right)$, the leading underdog will reduce $y_{j}$ compared to the $N E\left(y_{j}^{L}<y_{j}^{N}\right)$, which is retaliated by the follower with a reduction in $y_{i}\left(y_{i}^{F}<y_{i}^{N}\right)$. Note that $y_{j}^{L}<y_{j}^{N}<y_{j}^{\beta}$, where the last inequality stems from eq. (6). Thus, commitment by the weaker player does not induce a regime change with respect to the winning probability (or share): $p_{j}\left(\mathbf{y}^{N}\right)<p_{i}\left(\mathbf{y}^{N}\right) \Rightarrow p_{j}\left(\mathbf{y}^{S_{j}}\right)<p_{i}\left(\mathbf{y}^{S_{j}}\right)$. In particular, since $\varepsilon_{i j}<1, \mathbf{y}^{S_{j}}$ is even less balanced as $\mathbf{y}^{N}$, so that $y_{j}^{L} / y_{i}^{F}<y_{j}^{N} / y_{i}^{N}$ and therefore $p_{j}\left(\mathbf{y}^{S_{j}}\right)<p_{j}\left(\mathbf{y}^{N}\right)$. This case is 
represented in Figure 5 where player 1 is the favorite and 2 the underdog. In the case of a leading underdog the SPE $\left(\mathbf{y}^{S_{2}}\right)$ lies to the south-west of $\mathbf{y}^{N}$. Note that the effort reduction by both players makes the SPE Pareto dominate the simultaneous move NE. Also note that this finding is qualitatively similar with the finding by Dixit (1987) who showed that a leading underdog always undercommits effort.

Dixit (1987) also finds that a leading favorite always overcommits effort and that in case of an evenly matched contest $\left(c_{i}=c_{j}\right)$ there is no local incentive to precommit effort away from the simultaneous-move $N E$. We find that these results do not carry over to the case of DP. First, a

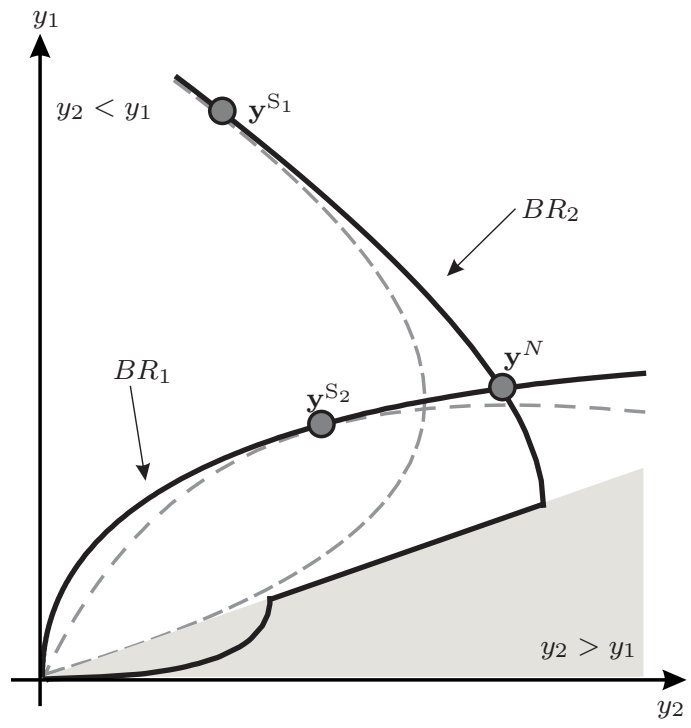

Figure 5

An overcommitting favorite and an undercommitting underdog

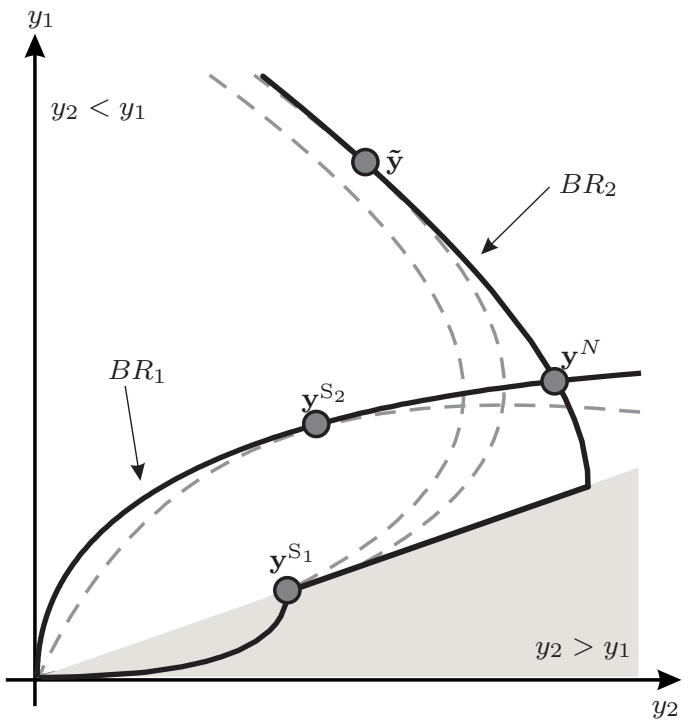

Figure 6

An undercommitting favorite and underdog

leading favorite overcommits effort in our model if and only if $\xi_{i}^{j}$ is above a certain threshold value (cf. proposition (4), case 2(a)). This case is also represented in Figure 5. If the favorite leads, the corresponding SPE $\left(\mathbf{y}^{S_{1}}\right)$ shows overcommitment by the leader $\left(y_{1}^{L}>y_{1}^{N}\right)$ and, since efforts are $S S$ for the underdog, a reduction of $y_{2}\left(y_{2}^{F}<y_{2}^{N}\right)$.

In case $\xi_{i}^{j}$ is below the above mentioned threshold, a leading favorite will undercommit effort in our model (see Proposition 4, case 2(b)). More precisely, the leader will choose $y_{j}^{L}=y_{j}^{\beta}$, which coincides with the minimum $y_{j}$ that is consistent with $y_{j}=B R_{i}\left(y_{j}\right)$. The intuition behind this result is the following: In case the favorite leads, he faces two counterbalancing effects: On the one hand an increase in $y_{j}$ beyond $y_{j}^{N}$ increases the equilibrium share compared to the NE, since efforts are $S S$ for the follower and thus $p_{j}\left(\mathbf{y}^{S}\right)>p_{j}\left(\mathbf{y}^{N}\right) \forall y_{j} \geq y_{j}$. On the other hand it increases the costs of effort. If the leader is inequality averse there is also an additional disutility from the resulting $A I$. A decrease in effort such that $y_{j}^{L}=y_{j}^{\beta}$ leads to a reduced equilibrium share compared to the NE, since $p_{j}\left(\mathbf{y}^{S}\right)=\frac{1}{2}<p_{j}\left(\mathbf{y}^{N}\right)$. However, it also minimizes the disutility from the leader's $A I$ aversion and reduces the costs of effort. Thus, if the leading favorite's $A I$ aversion is sufficiently strong or the marginal costs are large enough, the leader prefers the best possible 
egalitarian solution to any possible non-egalitarian one, $\tilde{\pi}_{j}\left(\underline{y}^{N}, \underline{y}^{N}\right)>\tilde{\pi}_{j}\left(y_{j}, B R_{i}\left(y_{j}\right)\right) \forall y_{j} \neq \underline{y}^{N}$. This case is represented in figure (6). Interestingly, this result does not depend on the fact that the follower is inequality averse, i.e. $y_{j}^{L}=\underline{y}^{N}$ may emerge even if $\beta_{i}>1$. This again shows that all results, whether in a simultaneous-move or sequential-move contest, crucially depend on both: The DI as well as AI parameter.

Furthermore, if $p_{i}\left(\mathbf{y}^{N}\right)=p_{j}\left(\mathbf{y}^{N}\right)=\frac{1}{2}$ we find that $y_{j}^{L}=y_{j}^{N}=y_{j}^{\beta}$ if $\xi_{i}^{j}=c_{j} / c_{i}$, i.e. if a unique egalitarian $N E$ exists. If $\xi_{j}^{i} \leq c_{i} / c_{j} \leq\left(\xi_{i}^{j}\right)^{-1}$ (with at least one inequality being strict) we find that $y_{j}^{L}=y_{j}^{\beta} \leq y_{j}^{N}$. Two possible cases have to be distinguished: If $y_{j}^{\beta} \geq y_{i}^{\beta}$, then the minimumeffort egalitarian $N E$ is given by $\underline{\mathbf{y}}^{N}=\left(y_{j}^{\beta}, y_{j}^{\beta}\right)$. Then, sequential moves serve as a coordination device and $\mathbf{y}^{S_{j}}=\underline{\mathbf{y}}^{N}$. In the opposite case $\left(y_{j}^{\beta}<y_{i}^{\beta}\right)$ the leader will even reduce effort beyond the minimum-effort egalitarian NE, since now $\underline{\mathbf{y}}^{N}=\left(y_{i}^{\beta}, y_{i}^{\beta}\right)$, so that $\mathbf{y}^{S_{j}}<\underline{\mathbf{y}}^{N}$.

\section{Summary and conclusions}

Our results show that distributional preferences of the Fehr and Schmidt (1999) type come a long way in explaining behavior in contests. This is true for share contests and even more so for probabilistic contests if it is assumed that individuals care for the ex-ante fairness of the contest mechanism. This finding is in contrast to the predictions of models with fairness preferences where individuals care for the ex post distribution of rents. In this latter case, the contest is isomorphic to a standard contest with asymmetric rents and therefore shares the behavioral predictions of an adequately designed model with selfish preferences. On the contrary, share contests or ex ante fairness concerns can lead to multiple equilibria in all situations where individuals are sufficiently similar with respect to their marginal effort costs, which helps to explain why overspreading is a common feature in experiments. Furthermore, if individuals care more for disadvantageous than for advantageous inequality, the set of overspending equilibria is relatively large compared to the set of underspending equilibria. Together with the finding by Bellemare, Kröger, and van Soest (2008) that especially well-educated young subjects care almost exclusively about disadvantageous inequality, this property can explain why overspending is a common phenomenon in experiments. Fairness concerns can also explain overdissipation in a rational-choice model because individuals get stuck in a situation where aggregate effort costs exceed the value of the prize because they want to avoid the emotional costs of disadvantageous inequality that would result if they invested less effort. This finding sheds a new light on the discussion about dissipation rates because it highlights the importance of the psychological costs of inequality. However, our model predicts that fairness preferences become behaviorally irrelevant in a simultaneous contest if either the number of players becomes large or if there exist selfish players.

Experimental tests of the contest model are rare to non existent if individuals differ in their abilities to compete. Given that our results for the case of homogenous players (with respect to their abilities) match the experimental evidence relatively closely, our results for the asymmetric case are of special relevance for future empirical tests. Two patterns became visible. First, distributional 
preferences often have an equalizing effect on the equilibrium. Second, this effect can be so large as to enforce a completely egalitarian outcome. In the extreme, it may even be the case that the less talented contestant wins a larger share (with higher probability) than his more talented opponent: lack of talent can be overcompensated by strong inequality aversion.

Strong inequality aversion, however, does not only play an interesting role in the simultaneousmove game. Sequential moves play a relatively obvious and a more subtle role for the behavior of players: coordination and commitment. In all cases where fairness concerns are sufficiently strong to induce a symmetric equilibrium in the simultaneous game, it is a Pareto improvement to let the less $A I$ averse player move first, irrespective of whether he is a favorite or underdog. In these cases, the leader's strategy coordinates expectations on a situation that would have been unattainable with simultaneous moves. But even if the more $A I$ adverse player moves first, sequential moves enable the players to solve the equilibrium-selection problem prevalent with simultaneous moves. Given that the set of symmetric equilibria can be Pareto ranked, it is clear that this coordination will be on the equilibrium that minimizes effort. But also in the case of asymmetric equilibria it may make sense to let the less $A I$ adverse player move first, because also in this case he can use the strong inequality aversion of the follower as a commitment device. 


\section{References}

Altmann, S., A. Falk, And M. Wibral (2012): "Promotions and Incentives: The Case of Multistage Elimination Tournaments," Journal of labor economics, 30(1), 149-174.

Anderson, S. P., J. K. Goeree, And C. A. Holt (1998): "Rent Seeking with Bounded Rationality: An Analysis of the All-Pay Auction," Journal of Political Economy, 106(4), 828-853.

Baharad, E., AND S. Nitzan (2008): "Contest Efforts in Light of Behavioural Considerations," The Economic Journal, 118, 2047-2059.

Baik, K. H., AND J. F. Shogren (1992): "Strategic Behavior in Contests: Comment," The American Economic Review, 82(1), 359-362.

Balafoutas, L., R. Kerschbamer, And M. Sutter (2012): "Distributional Preferences and Competitive Behavior," Journal of Economic Behavior \& Organization, 83(1), 125-135.

Bartling, B., E. Fehr, M. A. Maréchal, And D. Schunk (2009): "Egalitarianism and Competitiveness," The American Economic Review, 99(2), 93-98.

Baye, M. R., D. Kovenock, AND C. G. DE VRIES (1999): "The Incidence of Overdissipation in Rent-Seeking Contests," Public Choice, 99, 439-454.

- (2005): "Comparative Analysis of Litigation Systems: An Auction-Theoretic Approach," The Economic Journal, 115, 583-601.

- (2012): "Contests with Rank-Order Spillovers," Economic Theory, 51(2), 315-350.

Bellemare, C., S. Kröger, AND A. van Soest (2008): "Measuring Inequity Aversion in a Heterogeneous Population Using Experimental Decisions and Subjective Probabilities," Econometrica, 76(4), 815-839.

Besley, T., AND M. Ghatak (2008): "Status Incentives," The American Economic Review, 98(2), 206-211.

Blount, S. (1995): "When Social Outcomes Aren't Fair: The Effect of Causal Attributions on Preferences," Organizational Behavior and Human Decision Processes, 63(2), 131-144.

Brock, J. M., A. Lange, And E. Y. Ozbay (2010): "Dictating the Risk - Experimental Evidence on Giving in Risky Environments," The American Economic Review (fortcoming).

Charness, G., And D. I. Levine (2003): "The Road to Hell: An Experimental Study of Intentions," University of California at Santa Barbara, Economics Working Paper Series.

Charness, G., AND M. Rabin (2002): "Understanding Social Preferences With Simple Tests," The Quarterly Journal of Economics, 117(3), 817-869.

Chen, H., S. H. Ham, AND N. Lim (2011): "Designing Multiperson Tournaments with Asymmetric Contestants: An Experimental Study," Management Science, 57(5), 864-883.

Chowdhury, S. M., R. M. Sheremeta, And T. L. Turocy (2012): "Overdissipation and Convergence in Rentseeking Experiments: Cost Structure and Prize Allocation rules," Working Papers 12-13, Chapman University, Economic Science Institute.

Clark, D. J., AND C. Riss (1998): "Contest Success Functions: An Extension," Economic Theory, 11(1), 201-204.

Corchón, L. C. (2007): "The Theory of Contests: A Survey," Review of Economic Design, 11, 69-100.

Corchón, L. C., AND M. DAhM (2010): "Foundations for Contest Success Functions," Economic Theory, 43(1), $81-98$.

Cornes, R., And R. Hartley (2003): "Risk Aversion, Heterogeneity and Contests," Public Choice, 117, 1-25.

- (2005): "Asymmetric Contests with General Technologies," Economic Theory, 26, 923-946.

- (2012a): "Loss Aversion in Contests," The School of Economics Discussion Paper Series 1204, The University of Manchester.

- (2012b): "Risk Aversion in Symmetric and Asymmetric Contests," Economic Theory, 51(2), $247-275$.

Croson, R., AND A. ÖNÇÜLER (2005): "Strategic Rent-Seeking: An Experimental Investigation," Journal of Theoretical Politics, 17, 403-429.

Davis, D. D., AND R. J. Reilly (1998): "Do Too Many Cooks Always Spoil the Stew? An Experimental Analysis of Rent-Seeking and the Role of a Strategic Buyer," Public Choice, 95(1-2), 89-115.

Dechenaux, E., D. Kovenock, And R. M. Sheremeta (2012): "A Survey of Experimental Research on Contests, All-Pay Auctions and Tournaments," Working Paper.

Demougin, D., AND C. Fluet (2003): "Inequity Aversion in Tournaments," Centre interuniversitaire sur le risque, les politiques économiques et l'emploi, Cahier de recherche 03-22.

Dixit, A. (1987): "Strategic Behaviour in Contests," The American Economic Review, 77(5), 891-898.

Dubey, P., AND J. Geanakoplos (2010): "Grading Exams: 100, 99, 98,...or A, B, C?," Games and Economic Behavior, 69, 72-94.

Eisenkopf, G., AND S. Teyssier (2009): "Horizontal and Vertical Social Preferences in Tournaments," TWI Research Paper Series, 48.

(2010): "Envy and Loss Aversion in Tournaments," TWI Research Paper Series, 52.

FAlK, A., AND U. Fischbacher (2006): "A Theory of Reciprocity," Games and Economic Behavior, 54(2), 293-315.

FARWell, L., AND B. Weiner (1996): "Self-Perceptions of Fairness in Individual and Group Contexts," Personality and Social Psychology Bulletin, 22(9), 868-881.

Fehr, E., AND K. M. Schmidt (1999): "A Theory of Fairness, Competition, and Cooperation," The Quarterly Journal of Economics, 114(3), 817-868.

FonsecA, M. A. (2009): “An Experimental Investigation of Asymmetric Contests," International Journal of Industrial Organization, 27, 582-591. 
Gill, D., And R. Stone (2010): "Fairness and Desert in Tournaments," Games and Economic Behavior, 69(2), $346-364$.

Gradstein, M. (1995): "Intensity of Competition, Entry and Entry Deterrence in Rent Seeking Contests," Economics and politics, 7(1), 79-91.

Grund, C., AND D. SliwkA (2005): "Envy and Compassion in Tournaments," Journal of Economics and Management Strategy, 14(1), 187-207.

Herrmann, B., AND H. Orzen (2008): "The Appearance of Homo Rivalis: Social Preferences and the Nature of Rent Seeking," The Centre for Decision Research and Experimental Economics, School of Economics, University of Nottingham, 2008-10.

Hoffmann, M., And Grégoire Rota-Graziosi (2012): "Endogenous Timing in General Rent-Seeking and Conflict Models," Games and Economic Behavior, 75, 168-184.

Hoffmann, M., And M. Kolmar (2013): "Intention-Based Fairness Preferences in Two-Player Tullock Contests," CESifo working paper no. 4104.

Kagel, J. H., C. Kim, AND D. Moser (1996): "Fairness in Ultimatum Games with Asymmetric Information and Asymmetric Payoffs," Games and Economic Behavior, 13(1), 100-110.

Kahneman, D., AND D. T. Miller (1986): "Norm Theory: Comparing Reality to its Alternatives," Psychological Review, 93(2), 136-153.

Kong, X. (2008): "Loss Aversion and Rent-Seeking: An Experimental Study," The Centre for Decision Research and Experimental Economics, School of Economics, University of Nottingham, 2008-13.

Konow, J. (2003): "Which Is the Fairest One of All? A Positive Analysis of Justice Theories," Journal of Economic Literature, 41(4), 1188-1239.

Konrad, K. A. (2009): Strategy and Dynamics in Contests. Oxford University Press, Oxford and New York.

KRÄKEL, M. (2008): "Emotions in tournaments," Journal of Economic Behavior ES Organization, 67(1), $204-214$.

KraWczyK, M. W. (2011): "A Model of Procedural and Distributive Fairness," Theory and Decision, 70(1), 111128.

LazeAr, E. P., AND S. Rosen (1981): "Rank-Order Tournaments as Optimum Labor Contracts," The Journal of Political Economy, 89(5), 841-864.

Leininger, W. (1993): "More Efficient Rent-Seeking - A Münchhausen Solution," Public Choice, 75, 43-62.

Lim, N. (2010): "Social Loss Aversion and Optimal Contest Design," Journal of Marketing Research, 47, 777-787.

Lim, W., A. Matros, And T. L. Turocy (2012): "Bounded Rationality and Group Size in Tullock Contests: Experimental Evidence," Working Paper.

Loewenstein, G. F., L. Thompson, And M. H. Bazerman (1989): "Social Utility and Decision Making in Interpersonal Contexts," Journal of Personality and Social Psychology, 57(3), 426-441.

Lugovskyy, V., D. Puzzello, And S. Tucker (2010): "An Experimental Investigation of Overdissipation in the All Pay Auction," European Economic Review, 54(8), 974-997.

Matros, A., And D. Armanios (2009): "Tullock's Contest with Reimbursements," Public Choice, 141, 49-63.

Millner, E. L., AND M. D. Pratt (1989): "An Experimental Investigation of Efficient Rent-Seeking," Public Choice, 62, 139-151.

- (1991): "Risk Aversion and Rent-Seeking: An Extension and Some Experimental Evidence," Public Choice, 69, 81-92.

Moldovanu, B., A. Sela, And X. Shi (2007): "Contests for Status," The Journal of Political Economy, 115(2), 338-363.

NitZan, S. (1994): "Modelling Rent-Seeking Contests," European Journal of Political Economy, 10, 41-60.

Offerman, T. (2002): "Hurting Hurts more than Helping Helps," European Economic Review, 46(8), $1423-1437$.

Potters, J., C. G. D. VRIEs, AND F. VAN Winden (1998): "An Experimental Examination of Rational RentSeeking," European Journal of Political Economy, 14(4), 783-800.

Rabin, M. (1993): "Incorporating Fairness into Game Theory and Economics," The American Economic Review, $83(5), 1281-1302$.

SEBAld, A. (2010): "Attribution and Reciprocity," Games and Economic Behavior, 68(1), 339-352.

Sheremeta, R. M. (2011): "Contest Design: An Experimental Investigation," Economic Inquiry, 49(2), 573-590.

Sheremeta, R. M., AND S. Y. Wu (2012): "Testing Canonical Tournament Theory: On the Impact of Risk, Social Preferences and Utility Structure," IZA Discussion Papers 6304.

Siegel, R. (2009): "All-Pay Contests," Econometrica, 77, 71-92.

Skaperdas, S. (1996): "Contest Success Functions," Economic Theory, 7(2), 283-290.

Szidarovszky, F. (1997): "On the Existence and Uniqueness of Pure Nash Equilibrium in Rent-Seeking Games," Games and Economic Behavior, 18, 135-140.

Tullock, G. (1980): "Efficient Rent Seeking," in Towards a Theory of the Rent-Seeking Society, ed. by J. Buchanan, R. Tollison, and G. Tullock, pp. 97-112. A \& M University Press.

VAn't Wout, M., R. S. Kahn, A. G. Sanfey, And A. Aleman (2006): "Affective State and Decision-Making in the Ultimatum Game," Experimental Brain Research, 169(4), 564-568.

Wallace, M. D. (1971): "Power, Status, and International War," Journal of Peace Research, 8, 23-35.

Watson, A. (2008): Enduring the Great War: Combat, Morale and Collapse in the German and British Armies, 1914-1918. Cambridge: Cambridge University Press.

Wilkinson-Ryan, T., AND D. Small (2008): "Negotiating Divorce: Gender and the Behavioral Economics of Divorce Bargaining," Law and Inequality, 26, 109-132. 


\section{Appendix A. Appendix - Proofs}

\section{Appendix A.1. Proof of PROPOSITION 1}

At a $N E$ we either have $y_{i}^{N}>y_{j}^{N}$ or $y_{i}^{N}=y_{j}^{N}$.

- If $y_{i}^{N}>y_{j}^{N}$ a $\beta$-type player $i$ encounters an $\alpha$-type player $j$. Utilizing (6) then leads to

$$
y_{i}^{N}=\frac{\alpha_{j} \beta_{i}^{2} c_{j} V}{\left(\alpha_{j} c_{i}+\beta_{i} c_{j}\right)^{2}} \text { and } y_{j}^{N}=\frac{\alpha_{j}^{2} \beta_{i} c_{i} V}{\left(\alpha_{j} c_{i}+\beta_{i} c_{j}\right)^{2}},
$$

with

$$
y_{i}^{N}>y_{j}^{N} \Leftrightarrow \xi_{j}^{i}>\frac{c_{i}}{c_{j}} .
$$

Together with $\alpha_{i} \geq \beta_{i}$ and $\alpha_{j} \geq \beta_{j}$ we find the following condition for a unique $N E$ with $y_{i}^{N}>y_{j}^{N}$ :

$$
\left(\xi_{i}^{j}\right)^{-1}>\xi_{i}^{j}>\frac{c_{i}}{c_{j}}
$$

- In case $y_{i}^{N}=y_{j}^{N}$ we must have that $B R_{i}\left(y_{j}\right)=y_{j}$ and $B R_{j}\left(y_{i}\right)=y_{i}$ which, given eq. (6), only holds for

$$
\left[y_{j}^{\beta}, y_{j}^{\alpha}\right] \cap\left[y_{i}^{\beta}, y_{i}^{\alpha}\right]=\left[\underline{y}^{N}, \bar{y}^{N}\right] \text {, }
$$

with $\underline{y}^{N} \equiv \max \left\{y_{i}^{\beta}, y_{j}^{\beta}, 0\right\}$ and $\bar{y}^{N} \equiv \min \left\{y_{i}^{\alpha}, y_{j}^{\alpha}\right\}$, and which is non-empty if and only if

$$
\xi_{j}^{i} \leq \frac{c_{i}}{c_{j}} \leq\left(\xi_{i}^{j}\right)^{-1}
$$

Finally, notice that $\mathcal{N}(\mathbf{y})=\left\{y_{i}=y_{j} \mid \underline{y}^{N} \leq y_{i} \leq \bar{y}^{N}\right\}$ has mass one if and only if $\xi_{j}^{i}=\frac{c_{i}}{c_{j}}$.

\section{Appendix A.2. Proof of Proposition 2.A}

Because $f_{i}^{-1}\left(y_{i}\right)=c_{i} y_{i}$, we find that $x_{i}^{N}=c_{i} y_{i}^{N}$. The sum of efforts at a unique $N E\left(X^{N}=\sum_{i} x_{i}^{N}\right)$ is then given by

$$
X^{N}=\frac{c_{i} c_{j} \alpha_{j} \beta_{i}\left(\alpha_{i}+\beta_{j}\right)}{\left(c_{i} \alpha_{j}+c_{j} \beta_{i}\right)^{2}} V,
$$

whereas the sum of efforts in the selfish case is given by

$$
X^{*}=\left.X^{N}\right|_{\beta_{i}=1, \alpha_{j}=1}=\frac{2 c_{i} c_{j} V}{\left(c_{i}+c_{j}\right)^{2}} .
$$

Hence, $X^{N}>X^{*}$ if condition (17) holds and $X^{N}>V$ if condition (18) holds. In case $X^{N}>V$ we find that $\pi_{i}\left(\mathbf{y}^{N}\right)>0$ and $\pi_{j}\left(\mathbf{y}^{N}\right)<0$ if $\beta_{i} \leq 1$. If $\beta_{i}>1$ we may find that the opposite holds. Thus, despite the fact that the test for equilibrium is implicit in the proof of Proposition (1), the debate about the rationality of overdissipation (Baharad and Nitzan (2008)) justifies it to check explicitly that neither player can be better off by abstaining if the equilibrium implies overdissipation. Thus, we need to show that given $y_{i}^{N}\left(y_{j}^{N}\right)$ player $j(i)$ cannot gain by choosing $y_{j}=0\left(y_{i}=0\right)$. Indeed, supposing the opposite, we find that

$$
\pi_{i}\left(0, y_{j}^{N}\right)>\pi_{i}\left(\mathbf{y}^{N}\right) \Leftrightarrow \beta_{i}-\alpha_{i}>\frac{2 c_{j}^{2} \beta_{i}^{3}}{\left(c_{i} \alpha_{j}+c_{j} \beta_{i}\right)^{2}},
$$

and

$$
\pi_{j}\left(0, y_{i}^{N}\right)>\pi_{j}\left(\mathbf{y}^{N}\right) \Leftrightarrow 0>\frac{c_{i}^{2} \alpha_{j}^{3}}{\left(c_{i} \alpha_{j}+c_{j} \beta_{i}\right)^{2}},
$$

which obviously never holds. 


\section{Appendix A.3. Proof of Proposition 2.B}

Suppose $y_{i}^{\alpha}>y_{j}^{\alpha}$ so that $\frac{\alpha_{i}}{c_{i}}<\frac{\alpha_{j}}{c_{j}}$ and $y_{i}^{\beta}<y_{j}^{\beta}$ so that $\frac{\beta_{i}}{c_{i}}>\frac{\beta_{j}}{c_{j}}$. Then $y_{i}^{N}=y_{j}^{N} \in\left[\underline{y}^{N}, \bar{y}^{N}\right]$, with $\underline{y}^{N}=y_{j}^{\beta} \equiv \frac{\beta_{i} V}{4 c_{i}}$ and $\bar{y}^{N}=y_{j}^{\alpha} \equiv \frac{\alpha_{i} V}{4 c_{i}}$. The aggregate effort at $\overline{\mathbf{y}}^{N}=\left(\bar{y}^{N}, \bar{y}^{N}\right)$ is given by by $\bar{X}^{N}=c_{i} \bar{y}^{N}+c_{j} \bar{y}^{N}$. Then we find that

$$
\bar{X}^{N}>X^{*} \Leftrightarrow \frac{\alpha_{i} V\left(c_{i}+c_{j}\right)}{4 c_{i}}>\frac{2 c_{i} c_{j} V}{\left(c_{i}+c_{j}\right)^{2}} \Leftrightarrow \alpha_{i}>\frac{8 c_{i}^{2} c_{j}}{\left(c_{i}+c_{j}\right)^{3}} .
$$

and

$$
\bar{X}^{N}>V \Leftrightarrow \frac{\alpha_{i} V\left(c_{i}+c_{j}\right)}{4 c_{i}}>V \Leftrightarrow \alpha_{i}>\frac{4 c_{i}}{c_{i}+c_{j}} .
$$

The equilibrium utilities at $\overline{\mathbf{y}}^{N}$ are

$$
\pi_{i}\left(\overline{\mathbf{y}}^{N}\right)=\frac{\left(2-\alpha_{i}\right) V}{4} \text { and } \pi_{j}\left(\overline{\mathbf{y}}^{N}\right)=\frac{\left(2 c_{i}-\alpha_{i} c_{j}\right) V}{4 c_{i}} .
$$

The utilities in case that one of the players is abstaining from the contest are

$$
\pi_{i}\left(0, \bar{y}^{N}\right)=\frac{\left(1-\alpha_{i}\right) V}{2} \text { and } \pi_{j}\left(\bar{y}^{N}, 0\right)=\frac{\left(1-\alpha_{j}\right) V}{2},
$$

so that

$$
\pi_{i}\left(\overline{\mathbf{y}}^{N}\right)>\pi_{i}\left(0, \bar{y}^{N}\right) \Leftrightarrow 2 \alpha_{i} V>0
$$

and

$$
\pi_{j}\left(\overline{\mathbf{y}}^{N}\right)>\pi_{j}\left(\bar{y}^{N}, 0\right) \Leftrightarrow 2 V\left(2 \alpha_{j} c_{i}-\alpha_{i} c_{j}\right)>0,
$$

where the last inequality in (A.13) holds since $\alpha_{i} \geq 1$ (cf. assumption (2)), and in (A.15) since $y_{i}^{\alpha}>y_{j}^{\alpha}$ by assumption.

Aggregate effort at $\underline{\mathbf{y}}^{N}=\left(\underline{y}^{N}, \underline{y}^{N}\right)$ is given by $\underline{X}^{N}=c_{i} \underline{y}^{N}+c_{j} \underline{y}^{N}$. Consequently, substituting $\alpha_{i}$ by $\beta_{j}$ in eq. (A.10) as well as (A.11) leads to the corresponding conditions for overspending and overdissipation at $\underline{\mathbf{y}}^{N}$ and thus for all $\mathbf{y} \in \mathcal{N}(\mathbf{y})$

The equilibrium utilities at $\underline{\mathbf{y}}^{N}$ are

Since

$$
\pi_{i}\left(\underline{\mathbf{y}}^{N}\right)=\frac{\left(2-\beta_{i}\right) V}{4} \text { and } \pi_{j}\left(\underline{\mathbf{y}}^{N}\right)=\frac{\left(2 c_{i}-\beta_{i} c_{j}\right) V}{4 c_{i}} .
$$

(cf. eq. (A.13)) we find that

$$
\pi_{i}\left(0, \underline{y}^{N}\right)=\pi_{i}\left(0, \bar{y}^{N}\right) \text { and } \pi_{j}\left(\underline{y}^{N}, 0\right)=\pi_{j}\left(\bar{y}^{N}, 0\right),
$$

$$
\pi_{i}\left(\underline{\mathbf{y}}^{N}\right)>\pi_{i}\left(0, \underline{y}^{N}\right) \Leftrightarrow V\left(4 \alpha_{i}-2 \beta_{i}\right)>0
$$

and

$$
\pi_{j}\left(\underline{\mathbf{y}}^{N}\right)>\pi_{j}\left(\underline{y}^{N}, 0\right) \Leftrightarrow 2 V\left(2 \alpha_{j} c_{i}-\beta_{i} c_{j}\right)>0,
$$

where the last inequality in (A.18) holds since $\alpha_{i} \geq \beta_{i}$ and in (A.19) since $\frac{\beta_{i}}{\alpha_{j}}<\frac{c_{i}}{c_{j}}$ (cf. eq. (10), page 11).

\section{Appendix A.4. Proof of PROPOSITION 4}

The leader's utility is given by

$$
\tilde{\pi}_{j}\left(y_{j}\right)=\left\{\begin{array}{cc}
\frac{\alpha_{j} V y_{j}}{y_{j}+B R_{i}\left(y_{j}\right)}-\frac{\left(\alpha_{j}-1\right) V}{2}-c_{j} y_{j} & \text { for } \Delta \tilde{y}_{j}<0, \\
\frac{y_{j} V}{y_{j}+B R_{i}\left(y_{j}\right)}-c_{j} y_{j} & \text { for } \Delta \tilde{y}_{j}=0, \\
\frac{\beta_{j} V y_{j}}{y_{j}+B R_{i}\left(y_{j}\right)}+\frac{\left(1-\beta_{j}\right) V}{2}-c_{j} y_{j} & \text { for } \Delta \tilde{y}_{j}>0 .
\end{array}\right.
$$

It is easy to establish the strict concavity of $\tilde{\pi}_{j}\left(y_{j}\right)$ :

$$
\frac{d^{2} \tilde{\pi}_{j}\left(y_{j}\right)}{d y_{j}^{2}}=-\frac{1}{4 y_{j}} \sqrt{\frac{c_{i} V}{y_{j}}} \times\left\{\begin{array}{cl}
\frac{\alpha_{j}}{\sqrt{\beta_{i}}} & \text { for } \Delta \tilde{y}_{j}<0, \\
1 & \text { for } \Delta \tilde{y}_{j}=0, \\
\frac{\beta_{j}}{\sqrt{\alpha_{i}}} & \text { for } \Delta \tilde{y}_{j}>0 .
\end{array}\right.
$$

Thus, the first-order condition is necessary and sufficient for a maximum. The leader's marginal utility is given by equation (30). Given eq. (6) we get

$$
\frac{B R_{i}\left(y_{j}\right)-B R_{i}^{\prime}\left(y_{j}\right) y_{j}}{\left(y_{j}+B R_{i}\left(y_{j}\right)\right)^{2}}= \begin{cases}\sqrt{\frac{c_{i}}{4 \beta_{i} V y_{j}}}, & \text { for } y_{j}<y_{j}^{\beta}, \\ 0, & \text { for } y_{j} \in\left[y_{j}^{\beta}, y_{j}^{\alpha}\right], \\ \sqrt{\frac{c_{i}}{4 \alpha_{i} V y_{j}},}, & \text { for } y_{j} \in\left(y_{j}^{\alpha}, \hat{y}_{j}\right), \\ 0, & \text { for } y_{j} \geq \hat{y}_{j} .\end{cases}
$$


In the first interval $\left(y_{j}<y_{j}^{\beta}\right), B R_{i}\left(y_{j}\right)>y_{j}$, so that $B R_{i}\left(y_{j}\right) \equiv B R_{i}^{\beta}\left(y_{j}\right)$, in the third interval $\left(y_{j}>y_{j}^{\alpha}\right)$, $B R_{i}\left(y_{j}\right)<y_{j}$, so that $B R_{i}\left(y_{j}\right) \equiv B R_{i}^{\alpha}\left(y_{j}\right)$. In the second interval $\left(y_{j}^{\beta} \leq y_{j} \leq y_{j}^{\alpha}\right) B R_{i}\left(y_{j}\right)=y_{j}$, so that an increase in $y_{j}$ has no influence on the leader's equilibrium share. The same holds for the last interval $\left(y_{j} \geq \hat{y}_{j}\right)$, where $B R_{i}\left(y_{j}\right)=0$. Defining $y_{j}^{L} \equiv \underset{y_{j}}{\operatorname{argmax}} \tilde{\pi}_{j}\left(y_{j}\right)$ and $y_{i}^{F} \equiv B R_{i}\left(y_{j}^{L}\right)$, we find the following.

(I) $y_{j}<y_{j}^{\beta}$

In this case $y_{j}^{L}=y_{j}^{I} \equiv \frac{c_{i} V \alpha_{j}^{2}}{4 c_{j}^{2} \beta_{i}}$ and $y_{i}^{F}=y_{i}^{I} \equiv \frac{\alpha_{j} V\left(2 c_{j} \beta_{i}-\alpha_{j} c_{i}\right)}{4 c_{j}^{2} \beta_{i}}$. Note that $y_{j}^{I}<y_{i}^{I} \Leftrightarrow \xi_{j}^{i}>\frac{c_{i}}{c_{j}}$ so that $y_{i}^{N}>y_{j}^{N}$ (cf. corollary (2), page 13)). Moreover, $y_{j}^{I}<y_{j}^{N}, y_{i}^{I}>y_{i}^{N}$.

(II) $y_{j} \in\left[y_{j}^{\beta}, y_{j}^{\alpha}\right]$

Since $\frac{d \tilde{\pi}_{j}\left(y_{j}\right)}{d y_{j}}=-c_{j}<0$ in this interval, $y_{j}^{L}=y_{j}^{I I} \equiv \max \left\{0, y_{j}^{\beta}\right\} \leq y_{j}^{N}$ and $y_{i}^{F}=y_{j}^{I I} \leq y_{i}^{N}$.

(III) $y_{j} \in\left(y_{j}^{\alpha}, \hat{y}_{j}\right)$

In this case $y_{j}^{L}=y_{j}^{I I I} \equiv \frac{c_{i} \beta_{j}^{2} V}{4 c_{j}^{2} \alpha_{i}}$ and $y_{i}^{F}=y_{i}^{I I I} \equiv \frac{\beta_{j} V\left(2 \alpha_{i} c_{j}-\beta_{j} c_{i}\right)}{4 \alpha_{i} c_{j}^{2}}$. Note that $\hat{y}_{j}>y_{j}^{I I I}>y_{i}^{I I I} \Leftrightarrow \frac{1}{2} \xi_{i}^{j}<$ $\frac{c_{j}}{c_{i}}<\xi_{i}^{j}$ so that $y_{j}^{N}>y_{i}^{N}$. Moreover, $y_{j}^{I I I}>y_{j}^{N}, y_{i}^{I I I}<y_{i}^{N}$.

(IV) $y_{j} \geq \hat{y}_{j}$

Again, since $\frac{d \tilde{\pi}_{j}\left(y_{j}\right)}{d y_{j}}=-c_{j}<0, y_{j}^{L}=y_{j}^{I V} \equiv \hat{y}_{j}$ and $y_{i}^{F}=y_{i}^{I V} \equiv 0$. This only holds if $\Delta \equiv y_{j}^{N}-y_{i}^{N}$ is sufficiently large, i.e. if $\frac{1}{2} \xi_{i}^{j} \geq \frac{c_{j}}{c_{i}}$. Apparantley, $y_{j}^{I V}>y_{j}^{N}, 0=y_{i}^{I V}<y_{i}^{N}$.

Next, we check whether a leader in case $I, I I I$, or $I V$ can be better off by choosing $y_{j}^{I I}$.

1.

$$
\begin{aligned}
\pi_{j}\left(y_{j}^{I I}\right) & >\pi_{j}\left(y_{j}^{I}\right) \\
\Leftrightarrow \frac{V}{4}\left(2-\frac{\beta_{i} c_{j}}{c_{i}}\right) & >\frac{V}{4}\left(2+\alpha_{j}\left(\frac{\alpha_{j} c_{i}}{\beta_{i} c_{j}}-2\right)\right) \\
\Leftrightarrow 0 & >\left(\alpha_{j} c_{i}-\beta_{i} c_{j}\right)^{2} .
\end{aligned}
$$

Thus, if $y_{j}^{N}<y_{i}^{N} \Rightarrow y_{j}^{L}=y_{j}^{I} \wedge y_{i}^{F}=y_{i}^{I}$.

2. First, suppose that $\beta_{i}>0$ :

$$
\begin{aligned}
\pi^{j}\left(y_{j}^{I I}\right) & >\pi^{j}\left(y_{j}^{I I I}\right) \\
\Leftrightarrow \frac{V}{4}\left(2-\frac{\beta_{i} c_{j}}{c_{i}}\right) & >\frac{V}{4}\left(2+\beta_{j}\left(\frac{\beta_{j} c_{i}}{\alpha_{i} c_{j}}-2\right)\right) \\
\Leftrightarrow \alpha_{i} c_{j}^{2}\left(\alpha_{i}-\beta_{i}\right) & >\left(\beta_{j} c_{i}-\alpha_{i} c_{j}\right)^{2} .
\end{aligned}
$$

Thus, if $\xi_{i}^{j} \in\left(\frac{c_{j}}{c_{i}}, \frac{c_{j}}{c_{i}}\left(1+\sqrt{\frac{\alpha_{i}-\beta_{i}}{\alpha_{i}}}\right)\right) \Rightarrow y_{j}^{L}=y_{j}^{I I} \wedge y_{i}^{F}=y_{i}^{I I}$. For $\frac{\beta_{j}}{\alpha_{i}} \in\left(\frac{c_{j}}{c_{i}}\left(1+\sqrt{\frac{\alpha_{i}-\beta_{i}}{\alpha_{i}}}\right), \frac{2 c_{j}}{c_{i}}\right) \Rightarrow y_{j}^{L}=$ $y_{j}^{I I} \wedge y_{i}^{F}=y_{i}^{I I I}$. In case $\beta_{i}=0, \pi_{j}\left(y_{j}^{I I}\right)=\frac{V}{2}$ so that the inequality in (A.24) holds for all $\frac{\beta_{j}}{\alpha_{i}} \in\left(\frac{c_{j}}{c_{i}}, \frac{2 c_{j}}{c_{i}}\right)$. 3.

$$
\begin{aligned}
\pi_{j}\left(y_{j}^{I I}\right) & >\pi_{j}\left(y_{j}^{I V}\right) \\
\Leftrightarrow \frac{2 \beta_{j} c_{j}-\beta_{i} c_{i}}{\beta_{j} c_{j}} & >2 \\
\Leftrightarrow-\beta_{i} c_{i} & >0,
\end{aligned}
$$

which never holds. Thus, if $\xi_{i}^{j} \geq \frac{2 c_{j}}{c_{i}} \Rightarrow y_{j}^{L}=y_{j}^{I V} \wedge y_{i}^{F}=0$. 


\section{Appendix B. A contest with a general impact function}

We now turn to the general case, i.e. we make use of the general logit-type CSF introduced in assumption (1). In what follows, we will (for the sake of brevity) assume that $\beta_{i}>0$ and $\beta_{j}>0$, so that only non-trivial solutions are considered.

Because $\pi_{i}(\mathbf{y})$ (given by eq. (4), page (8)) is strictly concave and differentiable in $y_{i}$ the first order condition is necessary as well as sufficient for a best response:

$$
\frac{\partial \pi_{i}(\mathbf{y})}{\partial y_{i}} \leq 0
$$

In order to solve the general case it is now convenient to make use of the share-function approach, invented by Cornes and Hartley $(2003,2005) .{ }^{24}$ The following lemma describes the first-order conditions in case of advantageous and disadvantageous inequality.

\section{Lemma B.1}

Let $\sigma_{i}=y_{i} / Y$ be the share of total impact by individual $i$, and $Y=y_{i}+y_{j}$ be total impact. Then, under assumptions $(1)-(2)$, the FOCs in case of disadvantageous $\left(\Delta y_{i}<0\right)$ and advantageous $\left(\Delta y_{i}>0\right)$ inequality can be rewritten as:

$$
\begin{aligned}
& \alpha_{i}\left(1-\sigma_{i}\right) V-h_{i}^{\prime}\left(\sigma_{i} Y\right) Y \leq 0, \text { for } \alpha_{i} \bar{\sigma} V<h_{i}^{\prime}(\bar{\sigma} Y) Y, \\
& \beta_{i}\left(1-\sigma_{i}\right) V-h_{i}^{\prime}\left(\sigma_{i} Y\right) Y=0, \text { for } \beta_{i} \bar{\sigma} V>h_{i}^{\prime}(\bar{\sigma} Y) Y,
\end{aligned}
$$

with $\bar{\sigma}=\frac{1}{2}$ and equality at (B.2a) if $\sigma_{i}>0$

Proof.

The FOC in case of $D I$ is given by

$$
\frac{\alpha_{i}\left(1-\sigma_{i}\right) V}{Y}-h_{i}^{\prime}\left(\sigma_{i} Y\right) \leq 0 .
$$

Both terms in (B.3) are monotonic, with the first term strictly decreasing, and the second term non-decreasing in $\sigma_{i}$ and strictly positive for $\sigma_{i}>0$. If and only if

$$
\frac{\alpha_{i} \bar{\sigma} V}{Y}<h_{i}^{\prime}(\bar{\sigma} Y)
$$

there is some $\sigma_{i} \in[0, \bar{\sigma})$ which satisfies equation (B.3). Furthermore, if and only if $\frac{\alpha_{i} V}{Y}<h_{i}^{\prime}(0)$, the inequality in (B.3) holds.

In case of $A I$ the first-order condition is given by

$$
\frac{\beta_{i}\left(1-\sigma_{i}\right) V}{Y}-h_{i}^{\prime}\left(\sigma_{i} Y\right)=0,
$$

where, again, the first term is strictly decreasing, and the second term is non-decreasing in $\sigma_{i}$. Hence, if

$$
\frac{\beta_{i} \bar{\sigma}_{i} V}{Y}>h_{i}^{\prime}(\bar{\sigma} Y)
$$

then there is some $\sigma_{i}>\bar{\sigma}$ which satisfies equation (B.5).

Next, we turn to the above-mentioned share function and its properties.

\section{Lemma B.2 (Share function I)}

Given assumptions (1)-(2) a share function $s_{i}(Y)$ exists for player $i$. We find that $s_{i}(Y)=\sigma_{i}$, with $\sigma_{i}$ being either

(i) the unique solution to

$$
\alpha_{i}\left(1-\sigma_{i}\right) V-h_{i}^{\prime}\left(\sigma_{i} Y\right) Y=0
$$

if $\sigma \in(0, \bar{\sigma})$,

(ii) the unique solution to

$$
\beta_{i}\left(1-\sigma_{i}\right) V-h_{i}^{\prime}\left(\sigma_{i} Y\right) Y=0
$$

if $\sigma \in(\bar{\sigma}, 1]$,

\footnotetext{
${ }^{24}$ Originally, this approach was taken in order to solve the dimensionality problem associated with games characterized by a large number of players (see Cornes and Hartley, 2003, p. 2). Besides this virtue, the share-function approach has proven to be exceptionally usefull for our purpose, i.e. in an asymmetric two-player contest where players are endowed with distributional preferences.
} 
(iii) equal to $\bar{\sigma}$, if none of the above holds.

Proof. Omitted.

Next, we take a closer look at the properties of the share function.

\section{Lemma B.3 (Share function II)}

The share function $s_{i}(Y)$ has the following properties:

1. $\lim _{Y \rightarrow 0} s_{i}(Y)=1$,

2. $s_{i}^{\prime}(Y) \leq 0$ for $Y \geq 0$, with equality for $h_{i}^{\prime}(\bar{\sigma} Y) Y \in\left(\beta_{i} \bar{\sigma} V, \alpha_{i} \bar{\sigma} V\right)$,

3. $s_{i}(Y) \begin{cases}\in(0, \bar{\sigma}) & \text { for } h_{i}^{\prime}(\bar{\sigma} Y) Y>\alpha_{i} \bar{\sigma} V, \\ =\bar{\sigma} & \text { for } h_{i}^{\prime}(\bar{\sigma} Y) Y \in\left[\beta_{i} \bar{\sigma} V, \alpha_{i} \bar{\sigma} V\right], \\ \in(\bar{\sigma}, 1] & \text { for } h_{i}^{\prime}(\bar{\sigma} Y) Y<\beta_{i} \bar{\sigma} V,\end{cases}$

4. if $\lim _{y_{i} \rightarrow 0} h_{i}^{\prime}\left(y_{i}\right)=0$, then $s_{i}(Y)>0$ for $Y>0$, with $\lim _{Y \rightarrow \infty} s_{i}(Y)=0$. If not, $s_{i}(Y)=0$ for $Y \geq \bar{Y}$, with $\bar{Y}=\frac{\alpha_{i} V}{h_{i}^{\prime}(0)}$.

Proof.

(1) As $Y \rightarrow 0$, the left-hand side (LHS) of (B.6) goes to infinity while the right-hand side (RHS) goes to $h_{i}^{\prime}(0)$. Thus, for $Y$ small enough, $s_{i}(Y)>\bar{\sigma}$. Next, as $Y \rightarrow 0$, the first term of (B.5) goes to infinity while the second term goes to zero. Consequently $\lim _{Y \rightarrow 0} s_{i}(Y)=1$. (2) Since the first terms in (B.3) and (B.5) decrease in $Y$, while the marginal costs of impact are non-increasing in $Y, s_{i}(Y)$ is decreasing in $Y$ for $h_{i}^{\prime}(\bar{\sigma} Y) Y>\alpha_{i} \bar{\sigma} Y$ and $h_{i}^{\prime}(\bar{\sigma} Y) Y<\beta_{i} \bar{\sigma} Y$, i.e. for $s_{i}(Y) \neq \bar{\sigma}$. For $h_{i}^{\prime}(\bar{\sigma} Y) Y \in\left(\beta_{i} \bar{\sigma} V, \alpha_{i} \bar{\sigma} V\right)$ any marginal increase of $Y$ leaves $s_{i}(Y)=\bar{\sigma}$ unaffected. (3) Follows directly from (1) and (2). (4) If $h_{i}^{\prime}(0) \neq 0$, then there is always some $Y>0$ so that $\frac{\alpha_{i} \bar{\sigma} V}{Y} \leq h_{i}(0)$ and $s_{i}(Y)=0$. If $h_{i}(0)=0$ then $s_{i}(Y)>0$ for all $Y>0$.

Lemma B.3 shows that the share function is a strictly decreasing function of $Y$ if and only if $s_{i}(Y) \neq \bar{\sigma}$. Moreover, it shows that $s_{i}(Y)>0$ for all $Y \geq 0$ if $h_{i}(0)=0$ and thus $f_{i}(0) \rightarrow \infty$. This holds for example for the CSF introduced in Assumption (3) for $r<1$ (see Cornes and Hartley (2005) for an in depth discussion of this topic). For $h_{i}(0)>0$ there is always a $Y>0$ for which $s_{i}(Y)=0$.

Now we turn to the simoultaneous-move $N E$. A $N E$ exists if there is at least one $Y \geq 0$ for which the sum of agent's share functions (the aggregate share function $S(Y)=\sum_{i} s_{i}(Y)$ ) equals unity (see Cornes and Hartley (2003, 2005)). Lemma (B.3) together with the continuity of $s_{i}(Y)$ (and therefore of $S(Y)$ ) suffices to prove the existence of a NE.

Proposition B.1 (Cournot-Nash equilibrium)

Given assumptions (1)-(2) a simoultaneous-move $\mathrm{NE}\left(Y^{N}\right)$ exists. An egaliterian $\mathrm{NE}\left(s_{i}\left(Y^{N}\right)=s_{j}\left(Y^{N}\right)=\bar{\sigma}\right)$ exists if and only if

$$
h_{i}^{\prime}\left(\bar{\sigma} Y^{N}\right) Y^{N} \wedge h_{j}^{\prime}\left(\bar{\sigma} Y^{N}\right) Y^{N} \in\left[\beta_{i} \bar{\sigma} V, \alpha_{i} \bar{\sigma} V\right] \cap\left[\beta_{j} \bar{\sigma} V, \alpha_{j} \bar{\sigma} V\right]=\left[\max \left\{\beta_{i}, \beta_{j}\right\} \bar{\sigma} V, \min \left\{\alpha_{i}, \alpha_{j}\right\} \bar{\sigma} V\right] .
$$

The NE is unique if either

$$
h_{i}^{\prime}\left(\bar{\sigma} Y^{N}\right) Y^{N}=h_{j}^{\prime}\left(\bar{\sigma} Y^{N}\right) Y^{N}=\max \left\{\beta_{i}, \beta_{j}\right\} \bar{\sigma} V=\min \left\{\alpha_{i}, \alpha_{j}\right\} \bar{\sigma} V,
$$

or if the condition in (B.9) does not hold. In the opposite cases there is a continuum of equilbria.

Proof.

Existence of the $N E$ : A Nash equilibrium exists if $S(Y)=1$ for some $Y \geq 0$. Lemma (B.3.1), (B.3.2), (B.3.4) together with the continuity of $s_{i}(Y)$ (and therefore also of the aggregate share function $S(Y)$ ) suffices to prove the existence of a simoultaneous-move $N E$.

Egaliterian $N E$ : If $s_{i}\left(Y^{N}\right)=s_{j}\left(Y^{N}\right)=\bar{\sigma}$ then, given lemma (B.3.3), both conditions for $s_{i}\left(Y^{N}\right)=\bar{\sigma}$ and $s_{j}\left(Y^{N}\right)=$ $\bar{\sigma}$ must hold.

Uniqueness of the $N E$ : Since $\sum_{i} s_{i}^{\prime}(Y) \leq 0$ we can preclude the existence of isolated multiple equilibria. Therefore we either have a unique equilibrium or a continuum of equilibria. The former emerges if (i) $s_{i}^{\prime}\left(Y^{N}\right)<0$ and $s_{j}^{\prime}\left(Y^{N}\right)<0$, which only holds if $s_{i}\left(Y^{N}\right) \neq \bar{\sigma}$ and therefore $s_{j}\left(Y^{N}\right) \neq \bar{\sigma}$ as well, or (ii) if the intervall displayed in (B.9) becomes degenerate. Thus, a continuum emerges if and only if $S(Y)$ stays constant (and equal to 1 ) for some non-degenerate interval $I \subset \mathbb{R}_{+}$.

In general it is not possible to derive explicit functional forms of the share functions (see Cornes and Hartley (2003)). In order to derive explicitly the upper and lower bound of the continuum interval, we will, for the remainder of this 
appendix, assume that $f_{i}\left(x_{i}\right)=\frac{x_{i}^{r}}{c_{i}}$ with $r \leq 1$ and $c_{i}>0$, so that $h_{i}\left(y_{i}\right)=\left(c_{i} y_{i}\right)^{\frac{1}{r}}$. Then equating $h_{i}^{\prime}(\bar{\sigma} Y) Y$ to $\alpha_{i} \bar{\sigma} V\left(\beta_{i} \bar{\sigma}_{i} V\right)$ delivers the upper (lower) bound of the continuum interval (cf. eq. (B.4) and (B.6)):

$$
s_{i}(Y)=\bar{\sigma} \text { for } Y \in\left[\frac{\left(\beta_{i} r V\right)^{r} \bar{\sigma}^{(2 r-1)}}{c_{i}}, \frac{\left(\alpha_{i} r V\right)^{r} \bar{\sigma}^{(2 r-1)}}{c_{i}}\right] .
$$

Next, we take a closer look at two subcases: when players are asymmetric and when players are symmetric.

Appendix B.1. Asymmetric case

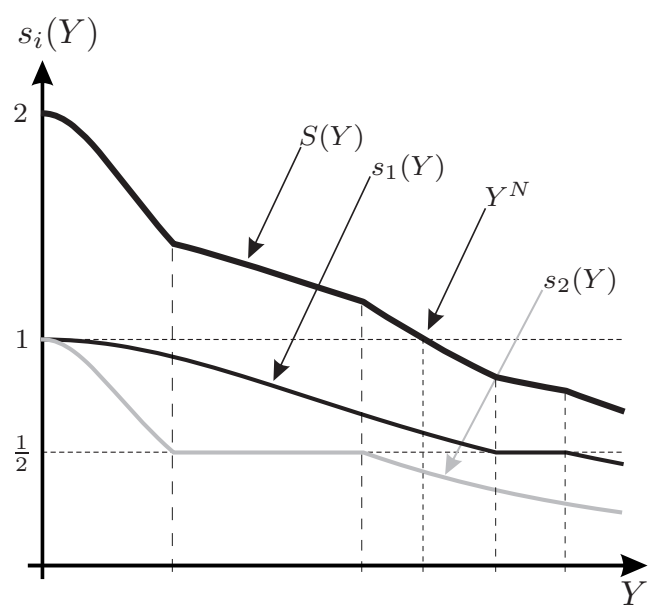

Figure B.1

Unique $N E$

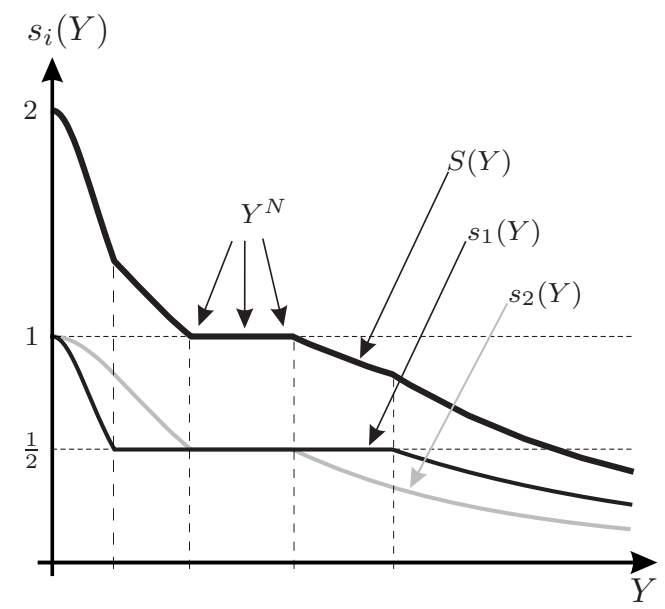

Figure B.2

Equilibrium correspondence

Suppose that $r=\frac{1}{2}$. Then the share function can be explicitly written as (cf. lemma (B.3.3))

$$
s_{i}(Y)= \begin{cases}\frac{\beta_{i} V}{2 c_{i}^{2} Y^{2}+\beta_{i} V} & \text { for } Y<\sqrt{\frac{\beta_{i} V}{2 c_{i}^{2}}}, \\ \bar{\sigma} & \text { for } Y \in\left[\sqrt{\frac{\beta_{i} V}{2 c_{i}^{2}}}, \sqrt{\frac{\alpha_{i} V}{2 c_{i}^{2}}}\right], \\ \frac{\alpha_{i} V}{2 c_{i}^{2} Y^{2}+\alpha_{i} V} & \text { for } Y>\sqrt{\frac{\alpha_{i} V}{2 c_{i}^{2}}} .\end{cases}
$$

Selfish preferences: In case of selfish preferences $\left(\alpha_{i}=\beta_{i}=\alpha_{j}=\beta_{j}=1\right)$ the $N E$ becomes

$$
Y^{*}=\sqrt{\frac{V}{2 c_{i} c_{j}}}, \text { with } s_{i}\left(Y^{*}\right)=\frac{c_{j}}{c_{j}+c_{i}}, \quad s_{i}\left(Y^{*}\right)=\frac{c_{i}}{c_{j}+c_{i}},
$$

so that

$$
y_{i}^{*}=\frac{1}{c_{i}+c_{j}} \sqrt{\frac{c_{j} V}{2 c_{i}}}, \quad y_{j}^{*}=\frac{1}{c_{i}+c_{j}} \sqrt{\frac{c_{i} V}{2 c_{j}}} .
$$

Apparently, player $i$ is the favorite (underdog) in the contest if and only if $c_{i}<c_{j}\left(c_{i}>c_{j}\right)$.

Distributional preferences: In case of $D P$, the $N E$ can be described as follows:

1. If $\left(\xi_{i}^{j}\right)^{-1} \geq \xi_{j}^{i} \geq\left(\frac{c_{i}}{c_{j}}\right)^{2}$ the game has a unique $N E$

$$
Y^{N}=\sqrt{\frac{V \sqrt{\alpha_{i} \beta_{j}}}{2 c_{i} c_{j}}}, \text { with } s_{i}\left(Y^{N}\right)=\frac{\sqrt{\alpha_{i}} c_{j}}{\sqrt{\alpha_{i}} c_{j}+\sqrt{\beta_{j}} c_{i}}, \quad s_{j}\left(Y^{N}\right)=\frac{\sqrt{\beta_{j}} c_{i}}{\sqrt{\alpha_{i}} c_{j}+\sqrt{\beta_{j}} c_{i}}
$$

so that

$$
y_{i}^{N}=\frac{\sqrt{c_{j} V} \alpha_{i}^{\frac{3}{4}} \beta_{j}^{\frac{1}{4}}}{\sqrt{2 c_{i}}\left(c_{j} \sqrt{\alpha_{i}}+c_{i} \sqrt{\beta_{j}}\right)}, \quad y_{j}^{N}=\frac{\sqrt{c_{i} V} \alpha_{i}^{\frac{1}{4}} \beta_{j}^{\frac{3}{4}}}{\sqrt{2 c_{j}}\left(c_{j} \sqrt{\alpha_{i}}+c_{i} \sqrt{\beta_{j}}\right)} .
$$


2. If $\xi_{j}^{i}<\left(\frac{c_{i}}{c_{j}}\right)^{2}<\left(\xi_{i}^{j}\right)^{-1}$ there is a continuum of equilibria, with

$$
Y^{N} \in\left[\max \left\{\sqrt{\frac{\beta_{i} V}{2 c_{i}^{2}}}, \sqrt{\frac{\beta_{j} V}{2 c_{j}^{2}}}\right\}, \min \left\{\sqrt{\frac{\alpha_{i} V}{2 c_{i}^{2}}}, \sqrt{\frac{\alpha_{j} V}{2 c_{j}^{2}}}\right\}\right], \quad y_{i}^{N}=y_{j}^{N}=\bar{\sigma} Y^{N} .
$$

Both cases are represented in figures (B.1) and (B.2), where $c_{2}>c_{1}$. In figure (B.1) we have $\xi_{2}^{1} \geq\left(\frac{c_{1}}{c_{2}}\right)^{2}$ so that the $N E$ is unique. Obviously, player 1 is the favorite because $s_{1}\left(Y^{N}\right)>s_{2}\left(Y^{N}\right)$. This is no longer the case in the second example, represented in figure (B.2). Here, we have a continuum of equilibria. Apparently, $\frac{\beta_{2}}{c_{2}^{2}}>\frac{\beta_{1}}{c_{1}^{2}}$ and $\frac{\alpha_{2}}{c_{2}^{2}}>\frac{\alpha_{1}}{c_{1}^{2}}$ so that the lower and upper bound of the equilibrium correspondence is determined by player 2 (cf. eq. (B.12)).

Next, we turn to the issues of overspending and overdissipation. For this, we need to determine aggregate effort in case of selfish preferences and DP. Utilizing the fact that $x_{i}=h_{i}\left(y_{i}\right)$ we find that aggregate effort at the selfish $N E$ $\left(X^{*}=x_{i}^{*}+x_{j}^{*}\right)$ equals:

$$
X^{*}=\left(c_{i} y_{i}^{*}\right)^{2}+\left(c_{j} y_{j}^{*}\right)^{2}=\frac{c_{i} c_{j} V}{\left(c_{i}+c_{j}\right)^{2}},
$$

whereas total effort in case of a unique $N E$ with DP is given by

$$
X^{N}=\left(c_{i} y_{i}^{N}\right)^{2}+\left(c_{j} y_{j}^{N}\right)^{2}=\frac{c_{i} c_{j} \sqrt{\alpha_{j} \beta_{i}}\left(\alpha_{j}+\beta_{i}\right) V}{2\left(c_{i} \sqrt{\alpha_{j}}+c_{j} \sqrt{\beta_{i}}\right)^{2}} .
$$

Proposition B.2 (Overspending and overdissipation in case of a unique NE)

Suppose $f_{i}\left(x_{i}\right)=\frac{\sqrt{x_{i}}}{c_{i}}$ so that $h_{i}\left(y_{i}\right)=\left(c_{i} y_{i}\right)^{2}$. Then we find that for $\left(\xi_{i}^{j}\right)^{-1} \geq \xi_{j}^{i} \geq \frac{c_{i}}{c_{j}}$ the following holds

$$
X^{N}>X^{*} \Leftrightarrow \frac{\sqrt{\alpha_{j} \beta_{i}}\left(\alpha_{j}+\beta_{i}\right)}{\left(\alpha_{j} c_{i}+\beta_{i} c_{j}\right)^{2}}>\frac{2}{\left(c_{i}+c_{j}\right)^{2}}
$$

and

$$
X^{N}>V \Leftrightarrow \beta_{i}+\alpha_{j}-4>2\left(\frac{c_{j}}{c_{i}} \sqrt{\xi_{j}^{i}}+\left(\frac{c_{j}}{c_{i}} \sqrt{\xi_{j}^{i}}\right)^{-1}\right)
$$

Proof.

The proof is similar to the proof of proposition (2.A), page (26) and is ommited here.

Since the RHS of (B.21) is minimized for $\xi_{j}^{i}=\left(\frac{c_{i}}{c_{j}}\right)^{2}$ we, as in the linear case, find that overdissiaption is the more likely to emerge the more equitable the contest is. However, since there are now diminishing returns to scale $(r<1)$ $D P \mathrm{~s}$ now have to play a greater role in order to evoke overdissipation. In particular, note that even if $\xi_{j}^{i}=\left(\frac{c_{i}}{c_{j}}\right)^{2}$, $\beta_{i}+\alpha_{j}>8$ in order to create overdissipation.

Proposition B.3 (Overspending and overdissipation in case of a continuum of NE) Suppose (without loss of generality) that $\frac{\beta_{i}}{c_{i}^{2}}>\frac{\beta_{j}}{c_{j}^{2}}$ and $\frac{\alpha_{i}}{c_{i}^{2}}<\frac{\alpha_{j}}{c_{j}^{2}}$. Under Assumptions (1) and (2) and for $\xi_{j}^{i}<$ $\left(\frac{c_{i}}{c_{j}}\right)^{2}<\left(\xi_{i}^{j}\right)^{-1}$ we find

$$
\left\{\begin{array}{l}
\text { probabilistic overspending } \\
\text { overspending }
\end{array}\right\}:=\left\{\begin{array}{l}
\left(c_{i} \bar{y}^{N}\right)^{2}+\left(c_{j} \bar{y}^{N}\right)^{2} \\
\left(c_{i} \underline{y}^{N}\right)^{2}+\left(c_{j} \underline{y}^{N}\right)^{2}
\end{array}\right\}>X^{*} \Leftrightarrow\left\{\begin{array}{c}
\alpha_{i} \\
\beta_{i}
\end{array}\right\}>\frac{8 c_{i}^{3} c_{j}}{\left(c_{i}^{2}+c_{j}^{2}\right)\left(c_{i}+c_{j}\right)^{2}},
$$

and

$$
\left\{\begin{array}{l}
\text { probabilistic overdissipation } \\
\text { overdissipation }
\end{array}\right\}:=\left\{\begin{array}{l}
\left(c_{i} \bar{y}^{N}\right)^{2}+\left(c_{j} \bar{y}^{N}\right)^{2} \\
\left(c_{i} \underline{y}^{N}\right)^{2}+\left(c_{j} \underline{y}^{N}\right)^{2}
\end{array}\right\}>V \Leftrightarrow\left\{\begin{array}{c}
\alpha_{i} \\
\beta_{i}
\end{array}\right\}>\frac{8 c_{i}^{2}}{c_{i}^{2}+c_{j}^{2}} .
$$

Proof. In case of a continuum of equilibria the upper bound and lower bound of the correspondence is determined according to (B.17). Thus, if $\frac{\beta_{i}}{c_{i}^{2}}>\frac{\beta_{j}}{c_{j}^{2}}$ and $\frac{\alpha_{i}}{c_{i}^{2}}<\frac{\alpha_{j}}{c_{j}^{2}}$, we find that

$$
\underline{y}^{N}=\sqrt{\frac{\beta_{i} V}{8 c_{i}^{2}}} \quad \text { and } \quad \bar{y}^{N}=\sqrt{\frac{\alpha_{i} V}{8 c_{i}^{2}}} .
$$


Then, aggregate effort at the lower bound of the equilibrium correspondence equals

$$
\underline{X}^{N}=\left(c_{i} \underline{y}^{N}\right)^{2}+\left(c_{j} \underline{y}^{N}\right)^{2}=\left(c_{i} \sqrt{\frac{\beta_{i} V}{8 c_{i}^{2}}}\right)^{2}+\left(c_{j} \sqrt{\frac{\beta_{i} V}{8 c_{i}^{2}}}\right)^{2}=\frac{\beta_{i} V}{8} \frac{c_{i}^{2}+c_{j}^{2}}{c_{i}^{2}},
$$

and aggregate effort at the upper bound equals

$$
\bar{X}^{N}=\left(c_{i} \bar{y}^{N}\right)^{2}+\left(c_{j} \bar{y}^{N}\right)^{2}=\left(c_{i} \sqrt{\frac{\alpha_{i} V}{8 c_{i}^{2}}}\right)^{2}+\left(c_{j} \sqrt{\frac{\alpha_{i} V}{8 c_{i}^{2}}}\right)^{2}=\frac{\alpha_{i} V}{8} \frac{c_{i}^{2}+c_{j}^{2}}{c_{i}^{2}} .
$$

Hence, if the upper bound of the equilbrium correspondecne is larger than $X^{*}(V)$ there is a positive probability of overspending (overdissipation), whereas if the lower bound of the equilbrium correspondecne is larger than $X^{*}(V)$, overspending (overdissipation) will emerge with proability 1.

\section{Appendix B.2. Symmetric case}

Suppose that $\alpha_{i}=\alpha_{j}=\alpha, \beta_{i}=\beta_{j}=\beta, c_{i}=c_{j}=c$ and $r \in(0,1]$.

Selfish preferences: In case of selfish preferences the unique and symmetric $N E$ becomes

$$
y_{i}^{*}=y_{j}^{*}=\left(\frac{r V}{4}\right)^{r} \frac{1}{c},
$$

so that aggregate effort at the selfish $N E$ equals

$$
X^{*}=2\left(c y^{*}\right)^{\frac{1}{r}}=\frac{r V}{2} .
$$

Distributional preferences: In case of DP $\left(\alpha>0\right.$ and $\left.\beta \in \mathbb{R}_{+}\right)$the symmetric $N E$ is never unique. The lower and upper bound of the equilibrium correspondence is given by (cf. eq. (B.11))

$$
\underline{y}^{N}=\frac{1}{c}\left(\frac{\beta_{i} r V}{4}\right)^{r} \quad \text { and } \quad \bar{y}^{N}=\frac{1}{c}\left(\frac{\alpha_{i} r V}{4}\right)^{r}
$$

Then, aggregate effort at the lower bound of the equilibrium correspondence equals

$$
\underline{X}^{N}=2\left(c \underline{y}^{N}\right)^{\frac{1}{r}}=\frac{r \beta V}{2} .
$$

and aggregate effort at the upper bound equals

$$
\bar{X}^{N}=2\left(c \bar{y}^{N}\right)^{\frac{1}{r}}=\frac{r \alpha V}{2} .
$$

Proposition B.4 (Overspending and overdissipation in case of a continuum of NE) Under Assumptions (1) and (2) and for $\alpha>1, \beta \in \mathbb{R}_{+}$we find

$$
\left\{\begin{array}{l}
\text { probabilistic overspending } \\
\text { overspending }
\end{array}\right\}:=\left\{\begin{array}{l}
2\left(c \bar{y}^{N}\right)^{\frac{1}{r}} \\
2\left(c \underline{y}^{N}\right)^{\frac{1}{r}}
\end{array}\right\}>X^{*} \Leftrightarrow\left\{\begin{array}{c}
\alpha \\
\beta
\end{array}\right\}>1,
$$

and

$$
\left\{\begin{array}{l}
\text { probabilistic overdissipation } \\
\text { overdissipation }
\end{array}\right\}:=\left\{\begin{array}{l}
2\left(c \bar{y}^{N}\right)^{\frac{1}{r}} \\
2\left(c \underline{y}^{N}\right)^{\frac{1}{r}}
\end{array}\right\}>V \Leftrightarrow\left\{\begin{array}{c}
\alpha \\
\beta
\end{array}\right\}>\frac{2}{r} .
$$

As in the case of a linear impact function, probabilistic overspending emerges in case of symmetric players if both are non-selfish $(\alpha>0)$, whereas overspending emerges with probability 1 is players are inequality prone $(\beta>1)$. Whether (probabilistic) overdissipation emerges at $\mathbf{y}^{N}$ now depends on the concavity of the impact function: The smaller $r$ the larger $\alpha(\beta)$ must be in order to find overdissipation (probabilistic overdissipation). 